bioRxiv preprint doi: https://doi.org/10.1101/2021.07.18.452807; this version posted July $19,2021$. The copyright holder for this preprint (which was not certified by peer review) is the author/funder, who has granted bioRxiv a license to display the preprint in perpetuity. It is made available under aCC-BY 4.0 International license.

\title{
Artificial Intelligence-rationalized balanced PPAR $\alpha / \gamma$ dual agonism resets the dysregulated macrophage processes in inflammatory bowel disease
}

Authors: Gajanan D. Katkar ${ }^{1}$, Ibrahim M. Sayed ${ }^{2 \$}$, Mahitha Shree Anandachar ${ }^{2}$, Vanessa Castillo ${ }^{1}$, Vidales, Eleadah ${ }^{1}$, Daniel Toobian ${ }^{1}$, Fatima Usmani ${ }^{2}$, Joseph R. Sawires ${ }^{3}$, Geoffray Leriche ${ }^{3}$, Jerry Yang ${ }^{3}$, William J. Sandborn ${ }^{4 *}$, Soumita Das ${ }^{2 *}$, Debashis Sahoo ${ }^{5,6,7 *}$ and Pradipta Ghosh ${ }^{1,4,7,8 *}$

\author{
Affiliations: \\ ${ }^{1}$ Department of Cellular and Molecular Medicine, University of California San Diego. \\ ${ }^{2}$ Department of Pathology, University of California San Diego. \\ ${ }^{3}$ Department of Chemistry and Biochemistry, University of California San Diego \\ ${ }^{4}$ Department of Medicine, University of California San Diego. \\ ${ }^{5}$ Department of Computer Science and Engineering, Jacob's School of Engineering, University of California San Diego. \\ ${ }^{6}$ Department of Pediatrics, University of California San Diego. \\ ${ }^{7}$ Rebecca and John Moore Comprehensive Cancer Center, University of California San Diego. \\ ${ }^{8}$ Veterans Affairs Medical Center, La Jolla, CA.
}

Alternative affiliation: ${ }^{\$}$ Department of Medical Microbiology and Immunology, Faculty of Medicine, Assiut University, Egypt.

Conflict of interest statement: S.D, D.S and P.G have a patent on the methodology. Barring this, the authors have declared that no conflict of interest exists.

Runnning title: AI-guided macrophage modulation in IBD

\section{*Correspondence to:}

William J. Sandborn, M.D.; Professor, Department of Medicine, University of California San Diego; 9500 Gilman Drive, MC 0956, La Jolla, CA 92093-0831.

Phone: 858-657-5331, Email: wsandborn@health.ucsd.edu

Soumita Das, Ph.D.; Associate Professor, Department of Pathology, University of California, San Diego; 9500 Gilman Drive, George E. Palade Bldg, Rm 256, 239; La Jolla, CA 92093.

Phone: 858-246-2062 (office): Email: sodas@ucsd.edu

Debashis Sahoo, Ph.D; Assistant Professor, Department of Pediatrics, University of California San Diego; 9500 Gilman Drive, MC 0730, Leichtag Building 132; La Jolla, CA 92093-0831.

Phone: 858-246-1803: Fax: 858-246-0019: Email: dsahoo@ucsd.edu

Pradipta Ghosh, M.D.; Professor, Departments of Medicine, and Cell and Molecular Medicine, University of California San Diego; 9500 Gilman Drive (MC 0651), George E. Palade Bldg, Rm 232, 239; La Jolla, CA 92093. Phone: 858-822-7633: Fax: 858-822-7636: Email: prghosh@ucsd.edu 
4 A computational platform, the Boolean network explorer $(B o N E)$, has recently been developed to infuse AI5 enhanced precision into drug discovery; it enables querying and navigating invariant Boolean Implication 6 Networks of disease maps for prioritizing high-value targets. Here we used BoNE to query an Inflammatory 7 Bowel Disease (IBD)-map and prioritize a therapeutic strategy that involves dual agonism of two nuclear 8 receptors, PPAR $\alpha / \gamma$. Balanced agonism of PPAR $\alpha / \gamma$ was predicted to modulate macrophage processes, 9 ameliorate colitis in network-prioritized animal models, 'reset' the gene expression network from disease to 0 health, and achieve a favorable therapeutic index that tracked other FDA-approved targets. Predictions were 1 validated using a balanced and potent PPAR $\alpha / \gamma$-dual agonist (PAR5359) in two pre-clinical murine models, 2 i.e., Citrobacter rodentium-induced infectious colitis and DSS-induced colitis. Using a combination of 3 selective inhibitors and agonists, we show that balanced dual agonism promotes bacterial clearance more 4 efficiently than individual agonists, both in vivo and in vitro. PPAR $\alpha$ is required and its agonism is sufficient 5 to induce the pro-inflammatory cytokines and cellular ROS, which are essential for bacterial clearance and 6 immunity, whereas PPAR $\gamma$-agonism blunts these responses, delays microbial clearance and induces the anti7 inflammatory cytokine, IL10; balanced dual agonism achieved controlled inflammation while protecting the 8 gut barrier and 'reversal' of the transcriptomic network. Furthermore, dual agonism reversed the defective 9 bacterial clearance observed in PBMCs derived from IBD patients. These findings not only deliver a 0 macrophage modulator for use as barrier-protective therapy in IBD, but also highlight the potential of BoNE 1 to rationalize combination therapy.

\section{KEY WORDS:}

6 Boolean Implication Network,

7 Inflammatory Bowel Disease (IBD),

8 Reactive Oxygen Species (ROS),

9 Macrophage,

0 Bacterial clearance,

1 Citrobacter,

2 PPAR $\alpha / \gamma-$ dual agonists

$3 \quad$ PAR5359 
Inflammatory bowel disease (IBD) is an autoimmune disorder of the gut in which diverse components including microbes, genetics, environment and immune cells interact in elusive ways to culminate in overt diseases (1-3). It is also heterogeneous with complex sub-disease phenotypes (i.e., strictures, fistula, abscesses and colitis-associated cancers $)(4,5)$. Currently, patients are offered anti-inflammatory agents that have a $\sim 30$ $40 \%$ response-rate, and $40 \%$ of responders become refractory to treatment within one year $(6,7)$. Little is known to fundamentally tackle the most widely recognized indicator/predictor of disease relapse i.e., a compromised mucosal barrier. Homeostasis within this mucosal barrier is maintained by our innate immune system, and either too little or too much reactivity to invasive commensal or pathogenic bacteria, is associated with $\operatorname{IBD}(8)$. Although defects in the resolution of intestinal inflammation have been attributed to altered monocyte-macrophage processes in IBD, macrophage modulators are yet to emerge as treatment modalities 7 in IBD (8).

We recently developed and validated an AI-guided drug discovery pipeline that uses large transcriptomic datasets (of the human colon) to build a Boolean network of gene clusters (9) (Figure 1; Step 0 ); this network differs from other computational methods (e.g., Bayesian and Differential Expression Analyses) because gene clusters here are interconnected by directed edges that represent Boolean Implication Relationships that invariably hold true in every dataset within the cohort. Once built, the network is queried using machine learning approaches to identify in an unbiased manner which clusters most effectively distinguish healthy from diseased samples and do so reproducibly across multiple other cohorts (906 human samples, 234 mouse samples). Gene-clusters that maintain the integrity of the mucosal barrier emerged as the genes that are invariably downregulated in IBD, whose pharmacologic augmentation/induction was predicted to 'reset' the network. These insights were exploited to prioritize one target, choose appropriate pre-clinical murine models for target validation and design patient-derived organoid models (Figure 1; Step 0) (9). Treatment efficacy was confirmed in patient-derived organoids using multivariate analyses. This AI-assisted approach provided a first-in-class epithelial barrier-protective agent in IBD and predicted Phase-III success with higher accuracy over traditional approaches (9).

Here we use the same AI-guided drug discovery pipeline to identify and validate a first-in-class macrophage modulator that is predicted to restore mucosal barrier and homeostasis in IBD (Figure 1; Steps 1). Using primary peritoneal macrophages and specific agonists and antagonists, we reveal the mechanism(s) of action that enable balanced agonists of this pair of nuclear receptors, PPAR $\alpha / \gamma$, to reverse some of the fundamental imbalances of the innate immune system in IBD, such that immunity can be achieved without overzealous inflammation (Figure 1; Step 2). We demonstrate the accuracy and predictive power of this network-rationalized approach and reveal the efficacy of balanced dual agonists of PPAR $\alpha / \gamma$ in two preclinical murine models (Figure; Step 3) and in patient-derived PBMCs (Figure; Step 4). 


\section{RESULTS}

2 Development of a web-based platform for generating a 'target report card'

3 We first developed an interactive, user-friendly web-based platform that allows the querying of our Boolean network-based-IBD map with the goal of enabling researchers to pick high-value targets (9) (Figure 1; Step 0; Supplemental Figure 1). The platform generates a comprehensive automated report containing actionable information for target validation, a 'target report card', which contains predictions on five components (Figure 2A): (i) Impact on the outcome of IBD in response to treatment, which shows how levels of expression of any proposed target gene(s) relates to the likelihood of response to therapies across diverse cohorts; (ii) Therapeutic index, a computationally generated index using Boolean implication statistics which provides a likelihood score of indicate whether pharmacologic manipulation of the target gene(s) would lead to success in Phase III clinical trials; (iii) Appropriateness of preclinical mouse models, a component that indicates which murine models of colitis shows the most significant change in the target genes (and hence, likely to be best models to test the efficacy of any manipulation of that target); (iv) Gender bias, a component that indicates whether the gene is differentially expressed in IBD-afflicted men versus women; and (v) Target tissue/cell type specificity, which shows the likely cell type where the target is maximally expressed, and hence, the cell type of desirable pharmacologic action. Details of how therapeutic index is computed are outlined in Methods and in Supplemental Figure 2; it is essentially a statistical score of how tightly any proposed target gene(s) associates with FDA-approved targets versus those that failed, and serves as an indicator of likelihood of success (9). Similarly, details of how cell type of action is computer are outlined in Methods and in Supplemental Figure 3.

\section{Ppar- $\alpha / \gamma$ dual agonists are predicted to be effective barrier-protective agents in IBD}

Previous work had identified a little over 900 genes in 3 clusters (Clusters \#1-2-3 within the IBD map; Figure 1: Step 0; Supplemental Figure 1A-B) as potentially high-value targets, all of which were invariably downregulated in IBD-afflicted colons (9). Reactome analyses showed that epithelial tight junctions (TJs), bioenergetics, and nuclear receptor pathway (PPAR signaling) related genes that are responsible for colon homeostasis are the major cellular processes regulated by these genes (Supplemental Figure 1B). Downregulation of genes in clusters \#1-3 was invariably associated also with an upregulation of genes in clusters \#4-5-6; reactome analyses of the latter showed cellular processes that concern immune cell activation, inflammation and fibrosis, which are hallmarks of IBD (Supplemental Figure 1B). Of the druggable candidates within C\#1-2-3, 17 targets were identified as associated with GO biological function of 'response to stress'/'response to stimuli'. Targeting one of the 17 targets, PRKAB1, the subunit of the heterotrimeric AMP-kinase engaged in cellular bioenergetics and stress response successfully restored the gut barrier function and also protected it from collapse in response to microbial challenge (9). Here, we prioritized two more of those 17 targets, PPARA and PPARG, which encode a pair of nuclear receptors, Ppar- $\alpha$ and Ppar- 

made available under aCC-BY 4.0 International license.

cluster \#2 and PPARG is in cluster \#3 (Supplemental Figure 1B). They both were located on the two major Boolean paths associated with epithelial barrier and inflammation/fibrosis (Supplemental Figure 1B)(9). Together, these findings imply three things: (i) that $P P A R A$ and $P P A R G$ are simultaneously downregulated in IBD, (ii) that such downregulation is invariably associated with inflammation, fibrosis and disruption of the epithelial barrier, and (iii) that simultaneous upregulation of $P P A R A$ and $P P A R G$ with agonists may restore the gut barrier. The last point is particularly important because Ppar $\alpha / \gamma$ agonists are known to augment the expression of PPARA and PPARG, and depletion of either reduced the expression of the other (10).

Noteworthy, while the role of Ppar- $\gamma$ in colitis has been investigated through numerous studies over the past 3 decades (11-13) (Supplemental Table 1), the role of Ppar- $\alpha$ has been contradictory (Supplemental Table 2), and their dual agonism in IBD has never been explored. All studies agree that Ppar- $\gamma$ agonists ameliorate DSS-induced colitis (13-15). Although claimed to be effective on diverse cell types in the gut (epithelium, T-cells, and macrophages), the most notable target cells of Ppar- $\gamma$ agonists are macrophages and dendritic cells (16). Furthermore, Phase I and II clinical trials with Ppar- $\gamma$ agonists either alone $(17,18)$ or in combination with mesalamine (19) show barrier protective effects in UC patients. Despite these insights, the biopharmaceutical industry has not been able to harness the beneficial impact of this major target within emergent therapeutic strategies largely due to a trail of withdrawals after devastating long-term side effects including heart failure, bone fracture, bladder cancer, fluid retention and weight gain $(20,21)$. Intriguingly, and of relevance to this work, the addition of Ppar- $\alpha$ agonistic activity to Ppar- $\gamma$, Ppar- $\gamma$, to Ppar- $\delta$ agonists have led to a higher safety profile, leading to their development for use in many diseases, including type 2 diabetes, dyslipidemia and non-alcoholic fatty liver disease (22).

\section{An automated target 'report card' for $P P A R A$ and $P P A R G$ in IBD}

We next generated an automated target report card for PPARA and PPARG. A high level of both PPARs, determined using a composite score for the abundance of both transcripts, was sufficient to distinguish healthy from IBD samples, not just in the test cohort that was used to build the IBD-map (ROC AUC of 0.74; Figure 2B; see also Supplemental Figure 2A-D), but also in four other independent cohorts with ROC AUC consistently above 0.88 (Figure 2C). High levels of both PPARs also separated responders from nonresponders receiving TNF $\alpha$-neutralizing mAbs, GSE16879, E-MTAB-7604 or Vedolizumab that block the $\alpha 4 \beta 7$ integrin to prevent selective gut inflammatory, GSE73661 (ROC AUC 0.63-0.89, Figure 2D), inactive disease from active disease (two independent cohorts ROC AUC above 0.93; Figure 2D), and quiescent UC that progressed, or not to neoplasia (ROC AUC=1.00 for qUC vs. nUC; Figure 2D). High level of PPARA and PPARG was also able to distinguish healthy from diseased samples in diverse murine models of colitis (Figure 2E); but such separation was most effectively noted in some models (Citrobacter infection-induced colitis, adoptive T-cell transfer, TNBS and $I L 10^{-/-}$), but not in others (DSS, and TNFR $1 / 2^{-/-}$). These findings imply that therapeutics targeting these two genes are best evaluated in the murine models that show the most consistent decrease in the gene expression, e.g., Citrobacter infection-induced colitis, adoptive T-cell transfer, 
4 TNBS, etc. This was intriguing because the majority ( $90 \%)$ of the published work on Ppara/g dual agonists 5 have been carried out in DSS models (Supplemental Table 1-2).

The expression profile of the target genes in the gut mucosa revealed that PPARA and PPARG are coexpressed at the highest levels in the crypt top epithelial cells and macrophages (Figure 2F; Supplemental

Figure 3), predicting that dual agonists are likely to preferentially act on these two cell types. The therapeutic 2E-F), aligned well with two other FDA-approved targets shown on the line graph (ITGB1, 0.046 and JAK2, 0.032). The index, which is a statistical measure of the strength of association of Ppar $\alpha / \gamma$ with genes that are targets of FDA-approved drugs that have successfully moved through the three phases of drug discovery (i.e., proven efficacy, with acceptable toxicity). A low number is indicative of a high likelihood of success in PhaseIII trials. Finally, PPARA and PPARG expression was downregulated to a similar extent in men and women with IBD (Figure $2 \mathrm{H}$ ), predicting that therapeutics targeting them are likely to be effective in both genders.

\section{Rationalization of $P P A R A / G$ and $P P A R G C 1 A$ as targets in IBD}

Because proteins, but not transcripts, are the targets of therapeutic agents, the impact of therapeutics is translated to cellular processes via protein-protein interaction (PPI) networks, a.k.a interactomes. We next asked how dual agonists of Ppar- $\alpha / \gamma$ might impact cellular pathways and processes. A PPI network visualized using Ppar- $\alpha$ and Ppar- $\gamma$ as 'query/input' and the interactive STRING v11.0 database (https://string-db.org/) as a web resource of known and predicted protein-protein interactions curated from numerous sources, including experimental data, computational prediction methods and public text collections. Pgcla (a product of the gene PPARGC1A) was a common interactor between the two PPARs (Figure 3A). We noted that Pgcla also happens to be a major component within the Ppar- $\alpha / \gamma$ functional network, serving as a central hub for positive feedback loops between the PPARs and their biological function (Figure 3B), i.e., mitochondrial biogenesis, DNA replication and energetics (electron transport chain and oxidative phosphorylation). When we analyzed the functional role of the interactomes of Ppar- $\alpha / \gamma$ we noted that indeed both interactomes converged on lipid metabolism, mitochondrial bioenergetics and circadian processes (Figure 3C), all representing major cellular processes that are known to be dysregulated in IBD (23-30). These findings are consistent with the finding that PPARA, PPARG and PPARGC1A are located within clusters \#1-2-3 and all of them are predicted to be progressively and simultaneously downregulated in IBD samples (Figure 3D; based on the IBD map, Supplemental Figure 1).

PPARA, PPARG and PPARGC1A are downregulated in Ulcerative colitis and Crohn's Disease

Previous work demonstrated that both Ppar- $\alpha$ and Ppar- $\gamma$ are highly expressed in the colon (31) and that their expression (proteins and mRNA) is downregulated (by $~ 60 \%$ ) in active UC (32), in both inflamed and noninflamed areas (33). Moreover, the expression of Ppar- $\gamma$ was significantly associated with disease activity (32). Polymorphisms have also been detected in Ppar- $\gamma$; while some studies found those to be associated with 

made available under aCC-BY 4.0 International license.

disease risk (35). We collected endoscopically obtained biopsies from the colons of healthy $(\mathrm{n}=7)$ and IBD ( $\mathrm{n}=14$ and 14 of $\mathrm{UC}$ and $\mathrm{CD}$, respectively) patients and assessed the levels of transcripts for PPARA, PPARG and PPARGC1A by qPCR (Figure 3E). We confirmed that all three transcripts were significantly downregulated in UC and $\mathrm{CD}$ samples compared to healthy; both PPARG and PPARGCla were more significantly downregulated in CD compared to UC (Figure 3F). These findings are in keeping with the network-based predictions that these genes should be downregulated invariably in all IBD samples, regardless of disease subtype (see individual disease maps; Supplemental Figure 4-5). While both PPARA and $P P A R G$ are in cluster \#2 in the UC map, PPARG and PPARA are in separate clusters, clusters 2 and 6, respectively, in the CD map (Supplemental Figure 4-5). Reactome pathway analyses implied that in the case of UC, the two nuclear receptors may co-regulate similar cellular homeostatic processes associated with cluster \#2, i.e., mitochondrial biogenesis and translation initiation, infectious disease and detoxification of ROS (see Supplemental Figure 4). By contrast, in the case of CD, they may independently regulate diverse cellular processes that maintain cellular homeostasis; while $P P A R G$ is associated with cellular metabolism (TCA cycle) and inhibition of NFkB signaling, PPARA is associated with transcriptional activity of nuclear receptors, cholesterol biosynthesis and Met/Ras signaling (see Supplemental Figure 5). Taken together, these findings demonstrate that PPARA/G and PPARGC1A are downregulated in IBD and that they may regulate key pathophysiologic processes that are vital for cellular homeostasis. Findings support our AI-guided hypothesis that restoration of the expression of these genes will increase the expression of genes in C\#1-2-3 and suppress the expression of, and that such increase.

\section{Synthesis and validation of PAR5359, a potent and specific Ppar- $\alpha / \gamma$ dual agonist}

We next sought to identify appropriate pharmacologic tools to test our hypothesis. Direct agonism of PPARGC1A/Pgcla was deemed as not feasible because the only known agonist, ZLN005, non-specifically and potently also activates AMPK (36), a target that is known to independently improve barrier integrity in IBD (9). Because Pgcla is intricately regulated by feedback loops by Ppar- $\alpha / \gamma$ (Figure 3B), we strategized targeting Pgc 1a indirectly via Ppar- $\alpha / \gamma$ instead. As for Ppar- $\alpha / \gamma$ dual agonists, we noted that all commercially available compounds lack 'balanced' agonistic activities (Supplemental Table 3) (37, 38). Drugs that have fallen aside due to safety concerns also lack balanced agonism; most of them are more potent on Ppar- $\gamma$ than on Ppar- $\alpha$ by a log-fold (Supplemental Table 3). All these Ppar- $\alpha / \gamma$ dual agonists have been withdrawn due to safety concerns (22), but the cause of the 'unsafe' profile remains poorly understood. Saroglitazar, the drug that is the only active ongoing Phase-III trial (NCT03061721) in this class, has $\sim 3$ log-fold more potency on Ppar- $\alpha$ than Ppar- $\gamma$ (39). Because our AI-guided approach suggested the use of simultaneous and balanced agonism, we favored the use of the only balanced and yet, specific Ppar- $\alpha / \gamma$ agonist described to date, PAR5359 (40, 41) (see Supplemental Table 4). In the absence of commercial sources or well-defined methods on how to synthesize this molecule, we generated PAR5359 in 4 synthetic steps (see details in Methods) and confirmed its specificity and the comparable agonistic activities using pure single Ppar- $\alpha$ [GW7647 (42)] 
or Ppar- $\gamma$ [Pioglitazone (43)] agonists as controls (Supplemental Figure 6). With these potent and specific compounds as tools, and their doses adjusted to achieve the same potency, we set out to validate the networkbased predictions using pre-clinical models.

\section{PAR5359 ameliorates $C$. rodentium-induced colitis, enhances bacterial clearance}

We next sought to assess the efficacy of individual and dual agonists of our compounds in murine pre-clinical models. Ppar- $\alpha / \gamma$ 's role (or the role of their agonists) in protecting the gut barrier has been evaluated primarily in DSS-induced colitis (Supplemental Table 1, 2). This model is more related to the UC patient pathology. However, BoNE prioritized other models over DSS, many of which accurately recapitulate the Ppar- $\alpha$ and Ppary-downregulation that is observed in the barrier-defect transcript signature in human IBD (Figure 2E). Among those, we chose $C$. rodentium-induced infectious colitis, a robust model to study mucosal immune responses in the gut and understand derailed host-pathogen interaction and dysbiosis, which is closely related with IBD, and more specifically, CD pathophysiology (44-46). This model is also known to emulate the bioenergetic dysbalance and mitochondrial dysfunction(47), both key cellular processes represented in C\#12-3 within the IBD map (Supplementary Figure 1). Furthermore, this model requires the balanced action of macrophages (a cell line predicted to be the preferred cell type target; Figure 2F) to promote bacterial clearance and healing (48).

Colitis was induced by oral gavage of $C$. rodentium and mice were treated daily with the drugs via the intraperitoneal route (see Figure 4A for workflow; Supplemental Figure 7A). The dose for each drug was chosen based on their $E C_{50}$ on their respective targets so as to achieve equipotent agonistic activities (Supplemental Figure 6). Fecal pellets of individual mice were collected to determine the number of live bacteria present in the stool. As anticipated, the bacterial burden in all mice increased from day 5 , reaching a peak on day 7, forming a plateau until day 11 before returning to pre-infection baseline by day 18 (Figure 4B). Compared against all other conditions, PAR5359-treated mice cleared the gut bacterial load significantly and rapidly (Figure 4B-D). Citrobacter infection was associated with significant epithelial damage and profuse infiltration of inflammatory cells and edema by day 7 (Supplemental Figure 7B) most of which resolved by day 18 (DMSO control; Figure 4E). Colons collected on day 7 showed that treatment with PAR5359 significantly reduced these findings when compared to vehicle (DMSO), PPAR $\alpha$ and PPAR $\gamma$ agonists alone (Supplemental Figure 7B). Unexpectedly, when we analyzed the colons on day 18, we noted persistent immune infiltrates in tissues in two treatment arms, pioglitazone and GW7647 (arrowheads; Figure 4E), but not in the vehicle control group, or those treated with PAR5359. These findings indicate that individual Ppar- $\alpha$ or Ppar- $\gamma$ agonists may either retard bacterial clearance and/or induce an overzealous amount of inflammation, but the balanced dual agonist (PAR5359) may have effectively cleared infection and resolved inflammation. PAR5359 also reduced spleen inflammation as evidenced by a decreased spleen weight and length compared to vehicle control (Supplemental Figure 7C-F). The spleens of mice treated with DMSO, Ppar- $\alpha$-alone agonist, GW7647 and Ppar- $\gamma$-alone agonist, Pioglitazone showed black- 
treated with Ppar- $\alpha$-alone agonist, GW7647, showed a significant increase in spleen length (Supplemental Figure 7D, 7F).

Taken together, these findings indicate that Ppar- $\alpha / \gamma$ dual agonist PAR5359 is superior in ameliorating C. rodentium-induced colitis than either Ppar- $\alpha$ or Ppar- $\gamma$ agonist used alone. Treatment with the dual, but not the single agonists hastened bacterial clearance, resolved inflammation, and induced healing.

$0 \quad$ PAR5359 resets the colonic gene expression changes induced by $C$. rodentium infection Pharmacologic augmentation of PPARA and PPARG was hypothesized to be sufficient to upregulate genes in C\#1-2-3, and restore the entire transcriptomic network to 'healthy' state via the invariant Boolean implication relationships between the genes/clusters. We asked if that was achieved. RNA sequencing (RNA-seq) studies were carried out on the C. rodentium-infected colons in each treatment group (Figure 4A). As expected, downregulation of genes in clusters \#1-2-3 of the IBD-map was significant in infected untreated (DMSO control) $v s$. uninfected controls, indicative of network shift from health towards disease, and treatment with PAR5359 resisted such shift (Figure 4F).

Pre-ranked gene set enrichment analyses (GSEA) based on pair-wise differential expression analysis showed that when compared to DMSO control, dual Ppar- $\alpha / \gamma$ agonism with PAR5359, but not individual agonists Pioglitazone or GW7647 was able to significantly preserve epithelial junction signatures (both tight and adherens junctions) and balance macrophage processes (compare Figure 4G with Supplemental Figure 8A). These findings are in keeping with the predictions that epithelial cells and macrophages maybe the primary cell type of action for dual Ppar- $\alpha / \gamma$ agonists. Comparison of all treatment cohorts against each other revealed that although both PAR5359 and Pioglitazone were superior to GW7647 in maintaining some epithelial processes (differentiation, tight junctions) and macrophage processes (Supplemental Figure 8BE), PAR5359 emerged as the only group that maintained homeostatic PPAR signaling in nature and extent as uninfected control (Supplemental Figure 8F).

Taken together, these findings suggest that dual agonists of Ppar- $\alpha / \gamma$ are sufficient to either resist network shift and/or reverse the disease network in the setting of colitis. They also offer clues suggestive of epithelial and macrophage processes, two key cellular components of innate immunity in the gut lining as major mechanisms. These transcriptome wide impacts suggest that Ppar- $\alpha / \gamma$ dual agonist PAR5359 is superior in restoring colon homeostasis in C. rodentium-induced colitis than either Ppar- $\alpha$ or Ppar- $\gamma$ agonist used alone.

\section{PAR5359 ameliorates DSS-induced colitis}

It is well known that no single mouse model recapitulates all the multifaceted complexities of IBD (49, 50). Because almost all studies evaluating Ppar- $\alpha / \gamma$-modulators have been performed on the DSS-induced colitis model (Supplemental Table 1-2), we asked whether the Ppar- $\alpha / \gamma$ dual agonist PAR5359 can ameliorate colitis in this model. Mice receive intrarectal DMSO vehicle control or PAR5359 while receiving DSS in their drinking water (Supplemental Figure 9A). Disease severity parameters, i.e., weight loss, disease activity index, 
shortening of the colon and histology score were significantly ameliorated in the PAR5359-treated group (Supplemental Figure 9A-E). These findings show that the Ppar- $\alpha / \gamma$-dual agonist, PAR5359, is also effective in DSS-induced colitis. It is noteworthy that the PAR5359 dual agonist offered protection in the DSS-model, because prior studies using the same model have demonstrated that Ppar- $\alpha$ agonists worsen $(51,52)$, and that the Ppar- $\gamma$ agonists ameliorate colitis (53-55) (see Supplemental Table 1-2).

PAR5359 promotes bacterial clearance with controlled production of ROS and inflammation in 7 peritoneal macrophages

Next we sought to study the mechanism of action of PAR5359, and the target cell type responsible for the superiority of dual agonism over single agonism. Our AI-guided approach predicted crypt top epithelium and macrophages as site of action (Figure 2F). Based on prior studies with single agonists in cell-specific KO mice (Supplemental Table 1) and the phenotypes observed in our animal models (Figure 4; Supplementary Figure 8-9), single Ppar- $\gamma$ agonism appears sufficient to protect the epithelium in chemical-induced colitis (dual agonism did not offer additional advantage). The advantage of dual agonism is apparent in the Citrobacter-colitis model, which most robustly recapitulates the paradoxical immune suppression in the setting of dysbiosis that is seen in IBD, and most prominently in $\mathrm{CD}(44,56,57)$. Because the intestinal macrophages, the known to be the initiators of immune response, are alternatively polarized in this model(58, 59), we hypothesized that balanced agonism may alter macrophage response to dysbiosis. To test this hypothesis, we incubated macrophages treated or not with the drugs and challenged them with CD-associated adherent invasive E. coli (AIEC)-LF82; this strain, originally isolated from a chronic ileal lesion from a CD patient (60). As for the source of macrophages, we isolated metabolically active primary murine peritoneal macrophages using Brewer thioglycolate medium using established protocols $(61,62)$. These macrophages are known to have high phagocytic activity (61) (Figure 5A). Thioglycolate-induced peritoneal macrophages (TG-PMs) were lysed, and viable intracellular bacteria were counted after plating on an agar plate. Pretreatment with $1 \mu \mathrm{M}$ PAR5359 and an equipotent amount of GW7647 (Ppar- $\alpha$ agonist) promoted bacterial clearance and reduced the bacterial burden when compared to vehicle control (Figure 5B). By contrast, pretreatment with Pioglitazone (Ppar- $\gamma$ agonist) inhibited bacterial clearance; notably, bacterial burden was significantly higher at both $3 \mathrm{~h}$ and $6 \mathrm{~h}$ after infection (Figure 5B). Reduced clearance of microbes in the latter was associated also with reduced cellular levels of reactive oxygen species (ROS) (Figure 5C); oxidative burst and induction of ROS is key component for effective bacterial killing $(63,64)$. PAR5359 did not interfere with the production of microbe-induced ROS, and the Ppar- $\alpha$ agonist (GW7647) was permissive to ROS induction (in fact, even induces it over bacteria-alone control) during initial time points after infection (Figure $5 C)$.

These patterns of microbial clearance and cellular ROS were associated also with the expression of 4 cytokines, as determined by qRT-PCR analyses (Figure 5D). As expected, infection of TG-PM with AIEC- 
the pro-inflammatory cytokines $I l 1 \beta, I l 6$ and $\operatorname{Tnf} \alpha$ (but not the anti-inflammatory cytokine, Il10) (Figure 5D). By contrast, the Ppar- $\gamma$ specific agonist pioglitazone significantly suppressed all the cytokines, while there was no effect of the Ppar- $\alpha$ specific agonist GW7647 (Figure 5D). ELISA studied on the supernatant media further confirmed these findings (Figure 5E), demonstrating that the effects in gene expression were also translated to the levels of secreted cytokine protein released by the macrophages in the supernatant.

It is noteworthy that for the most part, the qPCR (Figure 5D) and ELISA (Figure 5E) studies matched, except Il10; although pioglitazone appeared to suppress $I l 10$ mRNA, it did not suppress the levels of the IL10 protein, suggesting that Ppar- $\gamma$ agonist is sufficient for an overall anti-inflammatory phenotype. Similarly, although GW7647 appeared to not affect Il10 mRNA, it suppressed the levels of the IL10 protein, suggesting that Ppar- $\alpha$ agonist is sufficient for an overall pro-inflammatory phenotype. Similar findings were also observed in the case of another enteric pathogen, S. enterica, i.e., unlike the dual agonist, neither Ppar$\alpha$ nor Ppar- $\gamma$ agonist could enhance bacterial clearance with a modest induction of pro-inflammatory cytokines (significantly lower than control) and, yet, had no impact on anti-inflammatory IL10 production 9 (Supplemental Figure 10). inflammation, inhibiting ROS production and delaying bacterial clearance; (ii) Ppar- $\alpha$ agonism enhances the induction of inflammation and ROS, and promotes bacterial clearance; and (iii) Ppar- $\alpha / \gamma$ dual agonism strikes a somewhat balanced response. The latter suppresses proinflammatory cytokines without suppressing antiinflammatory cytokine 1110 , and is permissive to inflammation and ROS induction that is optimal and sufficient to promote bacterial clearance.

\section{Ppar- $\alpha$, but not Ppar- $\gamma$ is required for the induction of inflammatory cytokines and ROS}

To further dissect which nuclear receptors are responsible for the balanced actions of the dual agonist, we next used a set of highly specific and potent Ppar- $\alpha / \gamma$ inhibitors (Supplemental Table 4). We pre-treated TG-PMs with Ppar- $\alpha$ and Ppar- $\gamma$ inhibitors, either alone, or in combination, followed by stimulation with bacterial cell wall component LPS (Figure 6A). As expected, LPS induced the cellular levels of ROS (Figure 6B) and inflammatory cytokines (Figure 6C-D) in TG-PMs significantly higher than in untreated control cells. Inhibition of Ppar- $\alpha$ suppressed the induction of cellular ROS and inflammatory cytokines, both at the level of gene and protein levels (Figure 6B-D). By contrast, inhibition of Ppar- $\gamma$ did not interfere with either response (Figure 6B-D). Simultaneous inhibition of both Ppar- $\alpha$ and Ppar- $\gamma$ mimicked the cellular phenotypes in the presence of Ppar- $\alpha$ inhibitors (Figure 6B-D), indicating that inhibition of Ppar- $\alpha$ is sufficient to recapitulate the phenotype of dual inhibition. Taken together, these findings indicate that Ppar- $\alpha$ is required 8 for the proinflammatory response of macrophages. 

made available under aCC-BY 4.0 International license.

1 In search of a pre-clinical human model for testing drug efficacy, we next assessed microbial handling by

2 PBMCs derived from patients with IBD and compared them with that in age-matched healthy volunteers. We

3 enrolled both male and female patients and both CD and UC (Supplemental Table 5). Consecutive patients

4 presenting for routine care to the UC San Diego IBD clinic were enrolled into the study; the only exclusion

5 criteria were failure to obtain informed consent for the study or active infections and/or disease flare.

6 Peripheral blood collected in the clinic was freshly processed as outlined in Figure 7A to isolate PBMCs. Pre-

7 treatment for 30 min with vehicle or PAR5359 was followed by infection for 1h. Subsequently, the cells were

8 treated with gentamicin for $60 \mathrm{~min}$ to kill extracellular bacteria to assess intracellular bacterial burden at 1 and

$96 \mathrm{~h}$ after the gentamicin wash.

0 Two observations were made: First, CD but not UC patient-derived PBMCs when infected with AIEC-

1 LF82 showed an increased number of internalized viable bacteria when compared to healthy PBMCs (Figure

$27 \mathrm{~B}, 7 \mathrm{E})$, indicative of either defective clearance and/or increased permissiveness to bacterial replication within

3 the cells is limited to the CD. Second, pre-treatment with PAR5359 could improve clearance significantly

4 (Figure 7C-D, 7F-G). These results indicate that bacterial clearance is delayed in PBMCs of patients with

$5 \mathrm{CD}$ and that Ppar- $\alpha / \gamma$ dual agonism with PAR5359 can reverse that defect. The possibility that such reversal

6 could be due to any direct bacteriostatic/-cidal effect of PAR5359 agonist was ruled out (see bacterial viability

7 assay in Supplemental Figure 11). Our findings demonstrate that bacterial clearance is delayed primarily in

$8 \mathrm{CD}$ and not UC are in keeping with the fact that delayed bacterial clearance from inflamed tissues (up to $\sim 4-$

9 fold) is uniquely observed in CD (65). These findings are also in keeping with our own observation that the

0 downregulation of PPARG/PPARGC1A was more prominent in patients with CD (Figure 3E-F). In fact,

1 delayed clearance is one of the major reasons for persistent inflammation and disease progression among

2 patients with $\mathrm{CD}(65,66)$. 
Barrier-protection/restoration is the treatment endpoint for all clinical trials in IBD therapeutics; however, despite much success in the development of anti-inflammatory therapies $(7,67)$, barrier-protective therapeutics in IBD have been slow to emerge (68). Here we report the discovery of an effective barrierprotective therapeutic strategy in IBD identified using an AI-guided navigation framework (summarized in Figure 8). First, a network-based drug discovery approach (9) was used to identify, rationalize and validate dual and balanced agonism of Ppar- $\alpha / \gamma$ (but not one at a time) is necessary for therapeutic success. Second, we provided evidence in the form of proof-of-concept studies (in two different pre-clinical murine models) demonstrating that the simultaneous and balanced agonistic activation of the pair of PPARs as an effective barrier protective strategy in IBD. Third, we demonstrate that macrophages are one of the primary target cell type of this therapeutic strategy; dual agonist (but not single) was permissive to the induction of macrophage responses expected for optimal immunity without overzealous inflammation. There are three notable takeaways from this study, which are unexpected observations and/or insights that fill key knowledge gaps in the fields of - (a) network medicine, (b) IBD therapeutics and (c) macrophage biology.

First, with regard to network medicine, the AI-guided approach we used here differs from the current practice in three fundamental ways: 1) Unlike most studies that prioritize targets based on Differential Expression Analysis (DEA, or integrated DEA) or Bayesian approaches, target identification and prediction, this work was guided by a Boolean implication network of continuum states in human disease (9); 2) Instead of conventional approaches of trial-and-error, intuitive guess and/or knowledge-based prioritization of study models (animal or cell-type of action), target validation in network-rationalized animal and cell-type models that most accurately recapitulate the role of the target(s) during disease progression; 3) Inclusion of human pre-clinical model (patient-derived PBMCs) for target validation, inspiring the concept of Phase ' 0 ' trials that have the potential to personalize the choice of therapies. The combined synergy of these approaches validates a first-in-class macrophage modulator in addressing the broken gut barrier in IBD.

The impact of using such an approach is 4-fold: (i) Because the network approach used here relies on the fundamental invariant Boolean implication relationships between genes, and their patterns of changes in expression between healthy and IBD samples, such 'rule of invariant' implies that any given relationship and/or change in expression pattern annotated within the network must be fulfilled in every IBD patient. By that token, targets/drugs prioritized based on this network is expected to retain efficacy beyond inbred laboratory mice, into the heterogeneous patient cohorts in the clinic. (ii) This AI-guided approach not just helped compute pre-test probabilities of success ("Therapeutic Index"), but also helped pick models that are most insightful and appropriate to demonstrate therapeutic efficacy (e.g., Citrobacter rodentium infectioninduced colitis) and to pinpoint the cell type and mechanism of action (microbial clearance by macrophages). This is noteworthy because the conventional approach in studying PPARs has been limited to the use of DSSinduced colitis (see Supplemental Table 1-2), which has often given conflicting results (see Supplemental

Table 2). Ppar- $\gamma$ agonists works best for the UC patients, perhaps because it is a potent inhibitor of 
proinflammatory cytokines and, as shown before, protects the intestinal epithelium_(69). Our findings in the Citrobacter model imply that such single Ppar- $\gamma$ agonism may worsen the macrophage dysfunction that is observed in the setting of $\mathrm{CD}$, which is characterized by ineffective microbial clearance, insufficient proinflammatory response in the setting of luminal dysbiosis (28). In fact, without the use of the Citrobacter rodentium infectious colitis model, the deleterious effects of Ppar- $\gamma$ agonists would have been overlooked. (iii) Having a computational framework improves precision in target identification; it is because of the emergence of the two PPARs (alongside their positive feedback regulator, Pgcla) within our network, we rationalized their dual agonism as a preferred strategy (over single) and our experiments validated that prediction both in vivo and in vitro. This is noteworthy because conventional approaches have demonstrated a protective role of Ppar- $\gamma$ agonists and a conflicting (both protective and exacerbating) role of Ppar- $\alpha$ in IBD $(52,70-72)$; the advantage of dual agonism has neither been rationalized nor tested. (iv) The 'target report card', like the one shown here, is a project navigation tool that is geared to streamline decision-making (i.e., which genes, which animal models, which cell type/cellular process, what is the likelihood of success, etc.), which in turn should reduce attrition rates, waste and delays; the latter are well-recognized flaws in the current process of drug discovery.

Second, regarding IBD therapeutics, our studies demonstrate that single or unbalanced combinations of Ppar agonists are inferior to dual/balanced agonists. Conventional and reductionist approaches have inspired numerous studies with single Ppar agonists over the past decade (Supplemental Tables 1-2). However, given the devastating side effects of most single or unbalanced Ppar- $\alpha / \gamma$ agonists (Supplemental Table 3), translating to the clinic beyond a Phase II trial $(17,73,74)$ has not been realized. Because the therapeutic index for the dual Ppar- $\alpha / \gamma$ agonists matches that of other FDA-approved targets/drugs, it is predicted that barring unexpected side effects, dual Ppar agonists are likely to be effective as barrier-protective agents. As for side effects, we noted is that balanced Ppar- $\alpha / \gamma$ agonists are rare; while all dual Ppar- $\alpha / \gamma$ agonists that have been discontinued due to side effects happen to be either single (only Ppar- $\gamma$ ) or 'unbalanced' (Ppar$\gamma>>$ Ppar- $\alpha$ agonistic activity), the newer generation formulations that are currently in the clinical trial have a reversed agonistic potency (Ppar- $\alpha>>$ Ppar- $\gamma$ agonistic activity) (see Supplemental Table 3). Because macrophage responses require finetuning (discussed below), our studies show how unopposed agonism of either Ppar- $\gamma$ or Ppar- $\alpha$ is harmful and can impair/dysregulate the way macrophages respond when microbes breach past the gut barrier. It is possible that many of the side effects of the discontinued thiazolidinediones are due to their inability to achieve that 'optimal' spectrum of macrophage function.

Third, when it comes to macrophage biology, this work sheds some unexpected and previously unforeseen insights into the role of the PPARs in the regulation of macrophage processes. Extensively studied for over $\sim 3$ decades, PPARs are known to regulate macrophage activation in health and disease (75). Targeting PPARs as a host-directed treatment approach to infectious/inflammatory diseases appears to be a sound strategy because they regulate macrophage lipid metabolism, cholesterol efflux, inflammatory responses (ROS and cytokine production), apoptosis, and production of antimicrobial byproducts (76). We found that 
unopposed Ppar- $\gamma$ activation suppresses bacterial clearance and blunts the induction of proinflammatory (but not anti-inflammatory, IL10) cytokines and ROS in response to infection both in vivo and in vitro. In other words, and consistent with prior reports, Ppar- $\gamma$ activation suppressed inflammation at the cost of impairing immunity. Our findings are in keeping with the findings of a systematic review and meta-analysis of 13 longterm randomized controlled trials that involved 17,627 participants $(8,163$ receiving Ppar- $\gamma$ agonists and 9,464 receiving control drugs)(77). Long-term ( 1-5.5 y) use of Ppar- $\gamma$ agonists increases the risk of pneumonia or lower respiratory tract infection significantly, some of which result in hospitalization, disability, or death(77).

In the case of Ppar- $\alpha$, unopposed activation-induced ROS and proinflammatory cytokines and accelerated bacterial clearance. Inhibitor studies further confirmed that Ppar- $\alpha$ was required for these responses (Figure 6). These findings are in keeping with others' showing that Ppar- $\alpha$, but not Ppar- $\gamma$ is required for NADPHinduced ROS formation both in human and murine macrophages (78). Ppar- $\alpha$ agonists induce the expression of NADPH oxidase subunits p47(phox), p67phox, and gp91phox, which are all essential functional components of NADPH complex (78). Dual and balanced Ppar- $\alpha / \gamma$ agonism enhanced bacterial clearance with only a moderate induction of proinflammatory cytokines or ROS. Such a response ensures that the macrophage functions within a 'goldilocks' zone, mounting inflammation that is just sufficient for microbial clearance and immunity. In our analysis, the only other PPAR-related gene within the IBD network, i.e., Pgcla, and its role within the Ppar- $\alpha / \gamma$ axis suggests that the intricate network of forward feedback loops orchestrated by Pgcla may be critical for achieving the critical balance between immunity and inflammation, which is a key outcome of the dual Ppar- $\alpha / \gamma$ agonists.

Because previous studies using cell-specific gene depletion have indicated that the barrier-protective role of Ppar- $\gamma$ may be mediated via cells other than the macrophages (54), namely, the T cells (79) and the epithelial cells (80), it is possible that the dual Ppar- $\alpha / \gamma$ agonists also act on those cells, promoting bacterial clearance and balancing cellular bioenergetics, ROS and cytokine production, in manners similar to that we observe in macrophages.

Taken together, our study uses an unconventional approach to rationalize and validate the use of Ppar$\alpha / \gamma$ dual agonists as first-in-class barrier protective macrophage modulators in the management of IBD. The approach is powerful because it leverages the precision of mathematics (Boolean algebra of logic) and the fundamental invariant patterns in gene expression (Boolean Implications). The AI-navigated drug discovery approach defined here could serve as a blueprint for future studies not just in IBD, but in any other such complex chronic diseases. 
9 P. G., D.S., S.D. and G.D.K conceptualized, supervised, administered the project and acquired funding to 0 support it. G.D.K., I.M.S., M.S.A, E.V., F.U., J.R.S were involved in data curation and formal analysis. D.S. 1 developed computational modeling and software for analysis and performed all computational analysis. 2 G.D.K., I.M.S., M.S.A, E.V., F.U., conducted animal studies for colitis. G.D.K., I.M.S., M.S.A, E.V., F.U., 3 and S.D., performed cell and tissue analysis including qPCR, ROS, bacterial clearance, ELISA. D.T. assisted 4 G.D.K. for qPCR and analysis. J.R.S., G.L. and J.Y. prepared PAR5359 compound. W.J.S. provided key 5 resources for human subjects. D.S., G.D.K. and P.G. prepared figures for data visualization, wrote original 6 draft, reviewed and edited the manuscript. All co-authors approved the final version of the manuscript.

\section{ACKNOWLEDGMENTS}

0 We thank Dharanidhar Dang (UCSD) for comments and critiques during the preparation of the manuscript. 1 This work was supported by National Institutes for Health (NIH) grants R01-AI141630 (to PG), DK107585 2 (to SD). PG, SD, and DS were also supported by the Leona M. and Harry B. Helmsley Charitable Trust and 3 the NIH (UG3TR003355, UG3TR002968 and R01-AI55696). GDK was supported through The American 4 Association of Immunologists Intersect Fellowship Program for Computational Scientists and Immunologists. 5 J.S. acknowledges support from the Interfaces Training Grant at UCSD (NIH T32EB009380). Authors thank 6 to Lee Swanson, Courtney Tindle, Stella-Rita Ibeawuchi, Julian Tam and Madhubanti Mullick for their 7 comments, feedback and technical support. This manuscript includes data generated at the UC San 8 Diego Institute of Genomic Medicine (IGC) using an Illumina NovaSeq 6000 that was purchased with 9 funding from a National Institutes of Health SIG grant (\#S10 OD026929). Additionally, a P30 grant 0 (NIH/NIDDK, P30DK120515) subsidized the RNA Seq and histology work showcased here. 
1. Richard ML, and Sokol H. The gut mycobiota: insights into analysis, environmental interactions and role in gastrointestinal diseases. Nat Rev Gastroenterol Hepatol. 2019;16(6):331-45.

2. Schirmer M, Garner A, Vlamakis H, and Xavier RJ. Microbial genes and pathways in inflammatory bowel disease. Nat Rev Microbiol. 2019;17(8):497-511.

3. Lavelle A, and Sokol H. Gut microbiota-derived metabolites as key actors in inflammatory bowel disease. Nat Rev Gastroenterol Hepatol. 2020.

4. Furey TS, Sethupathy P, and Sheikh SZ. Redefining the IBDs using genome-scale molecular phenotyping. Nat Rev Gastroenterol Hepatol. 2019;16(5):296-311.

5. Olivera P, Danese S, Jay N, Natoli G, and Peyrin-Biroulet L. Big data in IBD: a look into the future. Nat Rev Gastroenterol Hepatol. 2019;16(5):312-21.

6. Moschen AR, Tilg H, and Raine T. IL-12, IL-23 and IL-17 in IBD: immunobiology and therapeutic targeting. Nat Rev Gastroenterol Hepatol. 2019;16(3):185-96.

7. Ahluwalia JP. Immunotherapy in inflammatory bowel disease. Med Clin North Am. 2012;96(3):52544, $\mathrm{x}$.

8. Na YR, Stakenborg M, Seok SH, and Matteoli G. Macrophages in intestinal inflammation and resolution: a potential therapeutic target in IBD. Nat Rev Gastroenterol Hepatol. 2019;16(9):531-43.

9. Sahoo D, Swanson L, Sayed IM, Katkar GD, Ibeawuchi SR, Mittal Y, et al. Artificial intelligence guided discovery of a barrier-protective therapy in inflammatory bowel disease. Nat Commun. 2021;12(1):4246.

10. Kim SH, Hong SH, Park YJ, Sung JH, Suh W, Lee KW, et al. MD001, a Novel Peroxisome Proliferator-activated Receptor $\alpha / \gamma$ Agonist, Improves Glucose and Lipid Metabolism. Sci Rep. 2019;9(1):1656.

11. Annese V, Rogai F, Settesoldi A, and Bagnoli S. PPARgamma in Inflammatory Bowel Disease. PPAR Res. 2012;2012:620839.

12. Auwerx J. Nuclear receptors. I. PPAR gamma in the gastrointestinal tract: gain or pain? Am J Physiol Gastrointest Liver Physiol. 2002;282(4):G581-5.

13. Dubuquoy L, Rousseaux C, Thuru X, Peyrin-Biroulet L, Romano O, Chavatte P, et al. PPARgamma as a new therapeutic target in inflammatory bowel diseases. Gut. 2006;55(9):1341-9.

14. Ramakers JD, Verstege MI, Thuijls G, Te Velde AA, Mensink RP, and Plat J. The PPARgamma agonist rosiglitazone impairs colonic inflammation in mice with experimental colitis. J Clin Immunol. 2007;27(3):275-83.

15. Vetuschi A, Pompili S, Gaudio E, Latella G, and Sferra R. PPAR-gamma with its anti-inflammatory and anti-fibrotic action could be an effective therapeutic target in IBD. Eur Rev Med Pharmacol Sci. 2018;22(24):8839-48.

16. Xia H, Chen L, Liu H, Sun Z, Yang W, Yang Y, et al. Protectin DX increases survival in a mouse model of sepsis by ameliorating inflammation and modulating macrophage phenotype. Sci Rep. 2017;7(1):99.

17. Lewis JD, Lichtenstein GR, Stein RB, Deren JJ, Judge TA, Fogt F, et al. An open-label trial of the PPAR-gamma ligand rosiglitazone for active ulcerative colitis. Am J Gastroenterol. 2001;96(12):3323-8.

18. Huang Y, Wang C, Tian X, Mao Y, Hou B, Sun Y, et al. Pioglitazone Attenuates Experimental ColitisAssociated Hyperalgesia through Improving the Intestinal Barrier Dysfunction. Inflammation. 2020.

19. Liang HL, and Ouyang Q. A clinical trial of combined use of rosiglitazone and 5-aminosalicylate for ulcerative colitis. World J Gastroenterol. 2008;14(1):114-9.

20. Ogawa S, Takeuchi K, and Ito S. Plasma BNP levels in the treatment of type 2 diabetes with pioglitazone. J Clin Endocrinol Metab. 2003;88(8):3993-6.

21. Liao HW, Saver JL, Wu YL, Chen TH, Lee M, and Ovbiagele B. Pioglitazone and cardiovascular outcomes in patients with insulin resistance, pre-diabetes and type 2 diabetes: a systematic review and meta-analysis. BMJ Open. 2017;7(1):e013927.

22. Cheng HS, Tan WR, Low ZS, Marvalim C, Lee JYH, and Tan NS. Exploration and Development of PPAR Modulators in Health and Disease: An Update of Clinical Evidence. Int J Mol Sci. 2019;20(20). 

made available under aCC-BY 4.0 International license.

23. Scoville EA, Allaman MM, Brown CT, Motley AK, Horst SN, Williams CS, et al. Alterations in Lipid, Amino Acid, and Energy Metabolism Distinguish Crohn's Disease from Ulcerative Colitis and Control Subjects by Serum Metabolomic Profiling. Metabolomics. 2018;14(1):17.

24. Lai Y, Xue J, Liu CW, Gao B, Chi L, Tu P, et al. Serum Metabolomics Identifies Altered Bioenergetics, Signaling Cascades in Parallel with Exposome Markers in Crohn's Disease. Molecules. 2019;24(3).

25. Novak EA, and Mollen KP. Mitochondrial dysfunction in inflammatory bowel disease. Front Cell Dev Biol. 2015;3:62.

26. Pagel R, Bär F, Schröder T, Sünderhauf A, Künstner A, Ibrahim SM, et al. Circadian rhythm disruption impairs tissue homeostasis and exacerbates chronic inflammation in the intestine. Faseb $j$. 2017;31(11):4707-19.

27. Ray K. Mitochondrial dysfunction in Crohn's disease. Nat Rev Gastroenterol Hepatol. 2020;17(5):260.

28. Smith AM, Rahman FZ, Hayee B, Graham SJ, Marks DJ, Sewell GW, et al. Disordered macrophage cytokine secretion underlies impaired acute inflammation and bacterial clearance in Crohn's disease. J Exp Med. 2009;206(9):1883-97.

29. Smith SA, Ogawa SA, Chau L, Whelan KA, Hamilton KE, Chen J, et al. Mitochondrial dysfunction in inflammatory bowel disease alters intestinal epithelial metabolism of hepatic acylcarnitines. $J$ Clin Invest. 2021;131(1).

30. Weintraub Y, Cohen S, Chapnik N, Ben-Tov A, Yerushalmy-Feler A, Dotan I, et al. Clock Gene Disruption Is an Initial Manifestation of Inflammatory Bowel Diseases. Clin Gastroenterol Hepatol. 2020;18(1):115-22.e1.

31. !!! INVALID CITATION !!! (34).

32. Dou X, Xiao J, Jin Z, and Zheng P. Peroxisome proliferator-activated receptor- $\gamma$ is downregulated in ulcerative colitis and is involved in experimental colitis-associated neoplasia. Oncol Lett. 2015;10(3):1259-66.

33. !!! INVALID CITATION !!! (33).

34. !!! INVALID CITATION !!! $(34,35)$.

35. Zhang ZF, Yang N, Zhao G, Zhu L, and Wang LX. Association between the Pro12Ala polymorphism of peroxisome proliferator-activated receptor gamma 2 and inflammatory bowel disease: a metaanalysis. PLoS One. 2012;7(1):e30551.

36. Zhang LN, Zhou HY, Fu YY, Li YY, Wu F, Gu M, et al. Novel small-molecule PGC-1 $\alpha$ transcriptional regulator with beneficial effects on diabetic db/db mice. Diabetes. 2013;62(4):1297-307.

37. Takada I, and Makishima M. Peroxisome proliferator-activated receptor agonists and antagonists: a patent review (2014-present). Expert Opin Ther Pat. 2020;30(1):1-13.

38. Mirza AZ, Althagafi, II, and Shamshad H. Role of PPAR receptor in different diseases and their ligands: Physiological importance and clinical implications. Eur J Med Chem. 2019;166:502-13.

39. Jain MR, Giri SR, Trivedi C, Bhoi B, Rath A, Vanage G, et al. Saroglitazar, a novel PPAR $\alpha / \gamma$ agonist with predominant PPAR $\alpha$ activity, shows lipid-lowering and insulin-sensitizing effects in preclinical models. Pharmacol Res Perspect. 2015;3(3):e00136.

40. Kim MK, Chae YN, Son MH, Kim SH, Kim JK, Moon HS, et al. PAR-5359, a well-balanced PPARalpha/gamma dual agonist, exhibits equivalent antidiabetic and hypolipidemic activities in vitro and in vivo. Eur J Pharmacol. 2008;595(1-3):119-25.

41. Kim DK, Park EJ, Jeong JH, Jeon SH, Kim EJ, Shim HJ, et al. Liquid chromatography-tandem mass spectrometry of a new PPARalpha/gamma dual agonist PAR-5359 in rat plasma. Arch Pharm Res. 2009;32(12):1743-8.

42. Brown PJ, Stuart LW, Hurley KP, Lewis MC, Winegar DA, Wilson JG, et al. Identification of a subtype selective human PPARalpha agonist through parallel-array synthesis. Bioorg Med Chem Lett. 2001;11(9):1225-7.

43. Information NCfB. PubChem Compound Summary for CID 4829, Pioglitazone. Accessed 2020 Dec. 1, 2020.

44. Koroleva EP, Halperin S, Gubernatorova EO, Macho-Fernandez E, Spencer CM, and Tumanov AV. Citrobacter rodentium-induced colitis: A robust model to study mucosal immune responses in the gut. J Immunol Methods. 2015;421:61-72. 
bioRxiv preprint doi: https://doi.org/10.1101/2021.07.18.452807; this version posted July $19,2021$. The copyright holder for this preprint (which was not certified by peer review) is the author/funder, who has granted bioRxiv a license to display the preprint in perpetuity. It is made available under aCC-BY 4.0 International license.

45. Bosman ES, Chan JM, Bhullar K, and Vallance BA. Investigation of Host and Pathogen Contributions to Infectious Colitis Using the Citrobacter rodentium Mouse Model of Infection. Methods Mol Biol. 2016;1422:225-41.

46. Bhinder G, Sham HP, Chan JM, Morampudi V, Jacobson K, and Vallance BA. The Citrobacter rodentium mouse model: studying pathogen and host contributions to infectious colitis. $J$ Vis Exp. 2013(72):e50222.

47. Ma C, Wickham ME, Guttman JA, Deng W, Walker J, Madsen KL, et al. Citrobacter rodentium infection causes both mitochondrial dysfunction and intestinal epithelial barrier disruption in vivo: role of mitochondrial associated protein (Map). Cell Microbiol. 2006;8(10):1669-86.

48. Krause P, Morris V, Greenbaum JA, Park Y, Bjoerheden U, Mikulski Z, et al. IL-10-producing intestinal macrophages prevent excessive antibacterial innate immunity by limiting IL-23 synthesis. Nat Commun. 2015;6:7055.

49. Jiminez JA, Uwiera TC, Douglas Inglis G, and Uwiera RR. Animal models to study acute and chronic intestinal inflammation in mammals. Gut Pathog. 2015;7:29.

50. Kozaiwa K, Sugawara K, Smith MF, Jr., Carl V, Yamschikov V, Belyea B, et al. Identification of a quantitative trait locus for ileitis in a spontaneous mouse model of Crohn's disease: SAMP1/YitFc. Gastroenterology. 2003;125(2):477-90.

51. Qi Y, Jiang C, Tanaka N, Krausz KW, Brocker CN, Fang ZZ, et al. PPARalpha-dependent exacerbation of experimental colitis by the hypolipidemic drug fenofibrate. Am J Physiol Gastrointest Liver Physiol. 2014;307(5):G564-73.

52. Gu X, Song Y, Chai Y, Lu F, Gonzalez FJ, Fan G, et al. GC-MS metabolomics on PPARalphadependent exacerbation of colitis. Mol Biosyst. 2015;11(5):1329-37.

53. Mohapatra SK, Guri AJ, Climent M, Vives C, Carbo A, Horne WT, et al. Immunoregulatory actions of epithelial cell PPAR gamma at the colonic mucosa of mice with experimental inflammatory bowel disease. PLoS One. 2010;5(4):e10215.

54. Hontecillas R, Horne WT, Climent M, Guri AJ, Evans C, Zhang Y, et al. Immunoregulatory mechanisms of macrophage PPAR- $\gamma$ in mice with experimental inflammatory bowel disease. Mucosal Immunol. 2011;4(3):304-13.

55. Guri AJ, Mohapatra SK, Horne WT, 2nd, Hontecillas R, and Bassaganya-Riera J. The role of T cell PPAR gamma in mice with experimental inflammatory bowel disease. BMC Gastroenterol. 2010;10:60.

56. Higgins LM, Frankel G, Douce G, Dougan G, and MacDonald TT. Citrobacter rodentium infection in mice elicits a mucosal Th1 cytokine response and lesions similar to those in murine inflammatory bowel disease. Infect Immun. 1999;67(6):3031-9.

57. Yan D, Wang X, Luo L, Cao X, and Ge B. Inhibition of TLR signaling by a bacterial protein containing immunoreceptor tyrosine-based inhibitory motifs. Nat Immunol. 2012;13(11):1063-71.

58. Liu F, Smith AD, Solano-Aguilar G, Wang TTY, Pham Q, Beshah E, et al. Mechanistic insights into the attenuation of intestinal inflammation and modulation of the gut microbiome by krill oil using in vitro and in vivo models. Microbiome. 2020;8(1):83.

59. Paynich ML, Jones-Burrage SE, and Knight KL. Exopolysaccharide from Bacillus subtilis Induces Anti-Inflammatory M2 Macrophages That Prevent $\mathrm{T}$ Cell-Mediated Disease. $J$ Immunol. 2017;198(7):2689-98.

60. Boudeau J, Glasser AL, Masseret E, Joly B, and Darfeuille-Michaud A. Invasive ability of an Escherichia coli strain isolated from the ileal mucosa of a patient with Crohn's disease. Infect Immun. 1999;67(9):4499-509.

61. Pavlou S, Wang L, Xu H, and Chen M. Higher phagocytic activity of thioglycollate-elicited peritoneal macrophages is related to metabolic status of the cells. J Inflamm (Lond). 2017;14:4.

62. Layoun A, Samba M, and Santos MM. Isolation of murine peritoneal macrophages to carry out gene expression analysis upon Toll-like receptors stimulation. J Vis Exp. 2015(98):e52749.

63. Tan HY, Wang N, Li S, Hong M, Wang X, and Feng Y. The Reactive Oxygen Species in Macrophage Polarization: Reflecting Its Dual Role in Progression and Treatment of Human Diseases. Oxid Med Cell Longev. 2016;2016:2795090.

64. Tan H-Y, Wang N, Li S, Hong M, Wang X, and Feng Y. The Reactive Oxygen Species in Macrophage Polarization: Reflecting Its Dual Role in Progression and Treatment of Human Diseases. Oxidative Medicine and Cellular Longevity. 2016;2016:2795090. 
65. Segal AW. Studies on patients establish Crohn's disease as a manifestation of impaired innate immunity. J Intern Med. 2019;286(4):373-88.

66. Baillie JK, Arner E, Daub C, De Hoon M, Itoh M, Kawaji H, et al. Analysis of the human monocytederived macrophage transcriptome and response to lipopolysaccharide provides new insights into genetic aetiology of inflammatory bowel disease. PLoS Genet. 2017;13(3):e1006641.

67. Quezada SM, McLean LP, and Cross RK. Adverse events in IBD therapy: the 2018 update. Expert Rev Gastroenterol Hepatol. 2018;12(12):1183-91.

68. Harris MS, Wichary J, Zadnik M, and Reinisch W. Competition for Clinical Trials in Inflammatory Bowel Diseases. Gastroenterology. 2019;157(6):1457-61.e2.

69. Hontecillas R, and Bassaganya-Riera J. Expression of PPAR $\gamma$ in intestinal epithelial cells is dispensable for the prevention of colitis by dietary abscisic acid. Espen j. 2012;7(5):e189-e95.

70. Zhou X, Cao L, Jiang C, Xie Y, Cheng X, Krausz KW, et al. PPARalpha-UGT axis activation represses intestinal FXR-FGF15 feedback signalling and exacerbates experimental colitis. Nat Commun. 2014;5:4573.

71. Manoharan I, Suryawanshi A, Hong Y, Ranganathan P, Shanmugam A, Ahmad S, et al. Homeostatic PPARalpha Signaling Limits Inflammatory Responses to Commensal Microbiota in the Intestine. $J$ Immunol. 2016;196(11):4739-49.

72. Azuma YT, Nishiyama K, Matsuo Y, Kuwamura M, Morioka A, Nakajima H, et al. PPARalpha contributes to colonic protection in mice with DSS-induced colitis. Int Immunopharmacol. 2010;10(10):1261-7.

73. Lewis JD, Lichtenstein GR, Deren JJ, Sands BE, Hanauer SB, Katz JA, et al. Rosiglitazone for active ulcerative colitis: a randomized placebo-controlled trial. Gastroenterology. 2008;134(3):688-95.

74. Pedersen G, and Brynskov J. Topical rosiglitazone treatment improves ulcerative colitis by restoring peroxisome proliferator-activated receptor-gamma activity. Am J Gastroenterol. 2010;105(7):1595603.

75. Chawla A. Control of macrophage activation and function by PPARs. Circ Res. 2010;106(10):155969.

76. Leopold Wager CM, Arnett E, and Schlesinger LS. Macrophage nuclear receptors: Emerging key players in infectious diseases. PLoS Pathog. 2019;15(3):e1007585.

77. Singh S, Loke YK, and Furberg CD. Long-term use of thiazolidinediones and the associated risk of pneumonia or lower respiratory tract infection: systematic review and meta-analysis. Thorax. 2011;66(5):383-8.

78. Teissier E, Nohara A, Chinetti G, Paumelle R, Cariou B, Fruchart JC, et al. Peroxisome proliferatoractivated receptor alpha induces NADPH oxidase activity in macrophages, leading to the generation of LDL with PPAR-alpha activation properties. Circ Res. 2004;95(12):1174-82.

79. Guri AJ, Evans NP, Hontecillas R, and Bassaganya-Riera J. T cell PPARgamma is required for the anti-inflammatory efficacy of abscisic acid against experimental IBD. $J$ Nutr Biochem. 2011;22(9):812-9.

80. Hontecillas R, and Bassaganya-Riera J. Expression of PPAR gamma in intestinal epithelial cells is dispensable for the prevention of colitis by dietary abscisic acid. Espen j. 2012;7(5):e189-e95. 
bioRxiv preprint doi: https://doi.org/10.1101/2021.07.18.452807; this version posted July $19,2021$. The copyright holder for this preprint (which was not certified by peer review) is the author/funder, who has granted bioRxiv a license to display the preprint in perpetuity. It is made available under aCC-BY 4.0 International license.

\section{Step 0- CREATION AND VALIDATION OF AN INFLAMMATORY BOWEL DISEASE (IBD) MAP}

Artificial Intelligence- guided Discovery of a Barrier-Protective Therapy in Inflammatory Bowel Disease PMID: 34253728

Target \#1 PRKAB1-agonist

\section{Target \#2}

PPAR $\alpha / \gamma$ dual agonism

\section{Step 1- GENERATION OF AN AUTOMATED TARGET TARGET REPORT CARD}

Al-enabled prediction of the likelihood of success in Phase III trials and other key aspects of target biology; these guide choice of model systems.

\section{Step 2- MECHANISM OF ACTION STUDIES IN VITRO}

Balanced dual agonists of PPAR $\alpha / \gamma$ (not single) most optimally balance the pro- and anti-inflammatory signaling and favors microbe clearance.
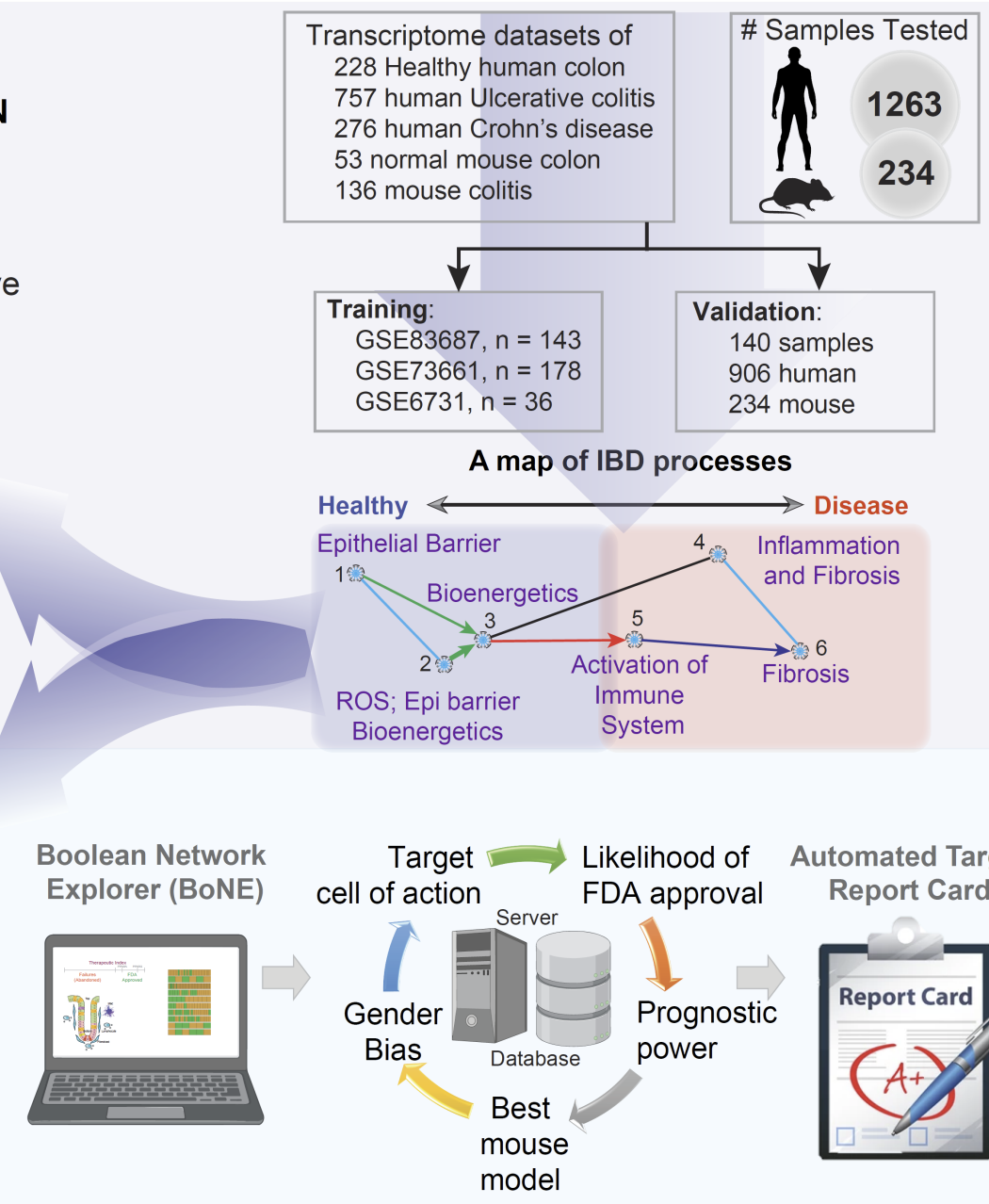

Automated Target Report Card

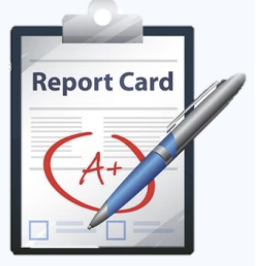

model

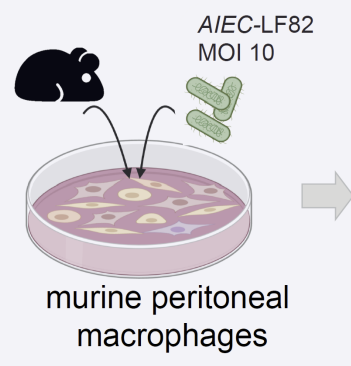

Inhibitors and Agonists of PPAR $\alpha$ PPAR $\gamma$ PPAR $\alpha / \gamma$ dual

Step 3- VALIDATION IN ANIMAL PRECLINICAL MODELS OF COLITIS

Dual agonists (not single) enhances microbe clearance, resets
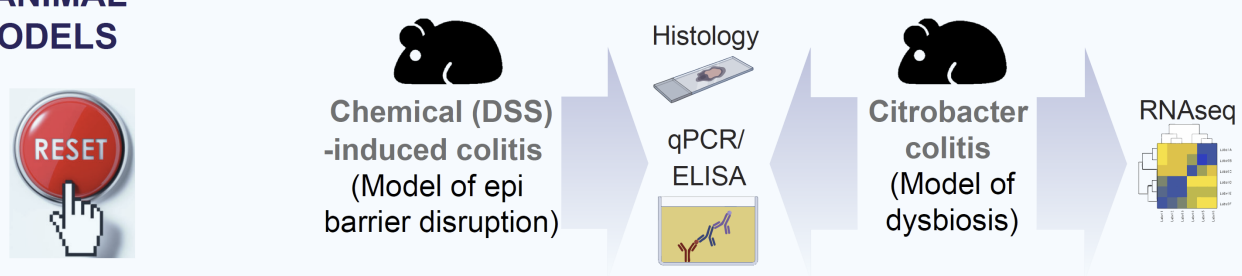
IBD network

\section{Step 4- VALIDATION IN HUMAN SUBJECTS}

Efficacy of bacterial clearance in peripheral blood derived monocytes

Figure 1. Study design. From top to bottom: The premise of a 4-step drug discovery pipeline is summarized on the top (Step 0) is a recently published(9) Boolean implication network-based computational model of disease continuum states 

made available under aCC-BY 4.0 International license.

1 in inflammatory bowel disease (IBD map). The map, comprised of 6 gene clusters, was created and validated database

2 containing 1497 gene-expression data (1263 human and 234 mouse samples). Paths, clusters and a list of genes in the 3 network-based model were prioritized to discover one clinically actionable drug target $(P R K A B 1)(9)$. Steps 1-4 outline 4 the AI-guided identification and validation of another target pair, PPARA and PPARG. Step 1: Dual agonists of $\$ \quad$ PPAR $\alpha / \gamma$ were predicted to - (i) modulate epithelial and macrophage processes; (ii) Citrobacter and chemical models 6 of colitis were predicted as most optimal models; (iii) have high therapeutic index indicative of likelihood to succeed in 7 Phase III clinical trials. Step 2: A combination of inhibitor and agonist studies helped establish that dual agonists reduce 8 inflammation (PPAR $\gamma$ ) while ensuring the induction of adequate immune response (PPAR $\alpha$ ). Step 3: Dural agonists 9 ameliorated colitis in two preclinical models of colitis, and reversed the patterns of disease-associated gene expression 0 that were altered in the IBD map. Step 4: In phase ' 0 ' human pre-clinical trials, PBMCs from CD, but not UC or healthy 1 showed defective microbe clearance; this defect was reversed with a dual agonist of PPAR $\alpha / \gamma$. 
bioRxiv preprint doi: https://doi.org/10.1101/2021.07.18.452807; this version posted July 19, 2021. The copyright holder for this preprint (which was not certified by peer review) is the author/funder, who has granted bioRxiv a license to display the preprint in perpetuity. It is made available under aCC-BY 4.0 International license.

A

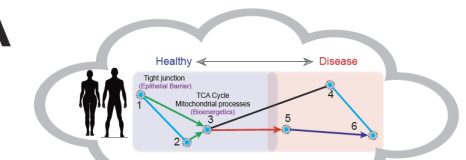

IBD map
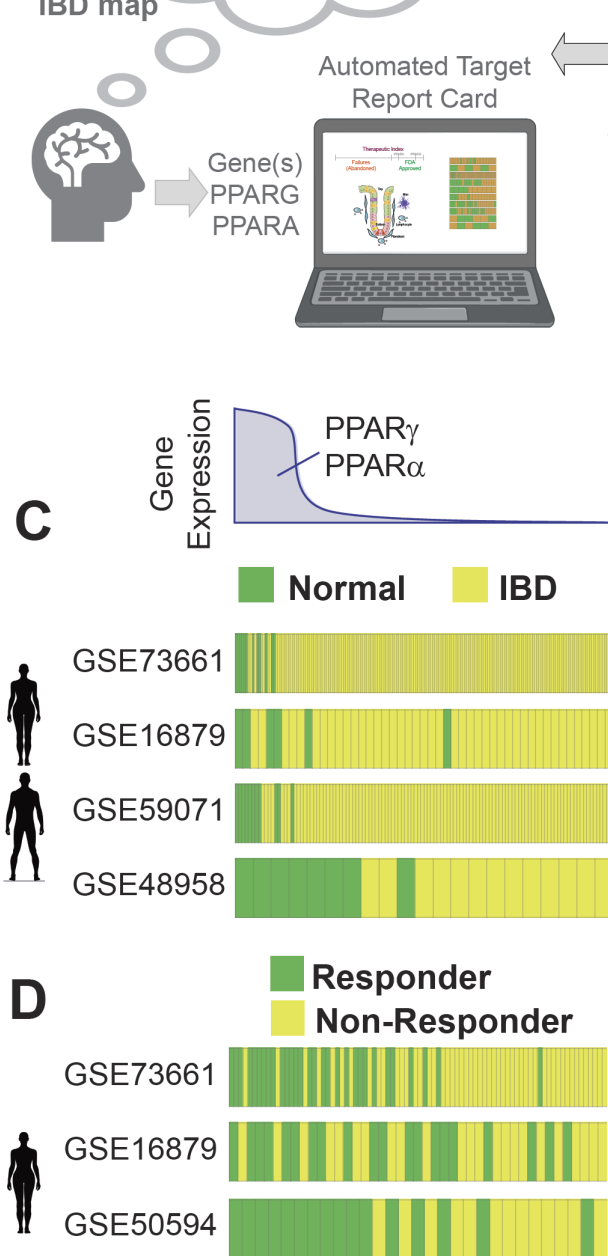

E-MTAB-7604

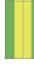

GSE59071
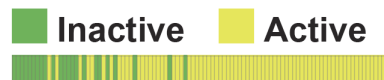

0.95

0.93

1.00

0.88

0.99

0.98

B

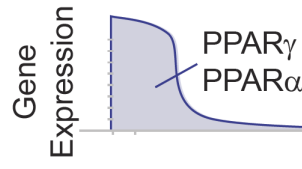

Normal UC $\quad C D$

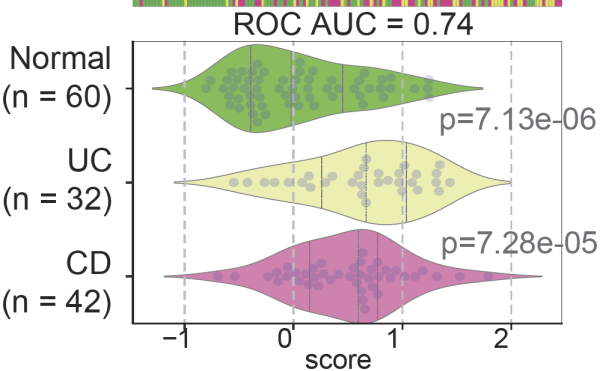

E

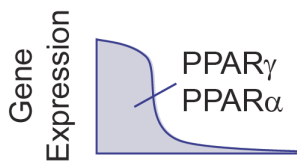

Mouse Model

DSS (bulk)

GSE42768

Normal Colitis $\mathrm{ROC}$

DSS (epithelium) E-MTAB-5249

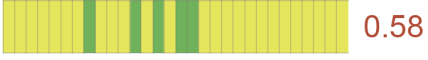

TNBS

GSE53835

Citrobacter

GSE90577

0.96

Adoptive T-cell

GSE87317

GSE27302

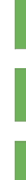

IL10 -/- (epithelium) GSE39859

IL10 -/- (bulk)

GSE39859

9

TNFR1 -/-

GSE107933

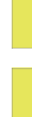

GSE65408

0.90

TNFR2 -/-

G

Therapeutic Index

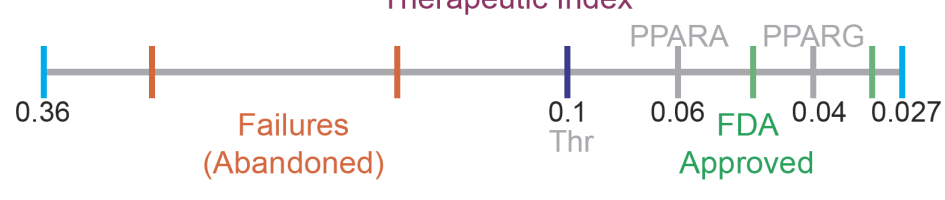

F

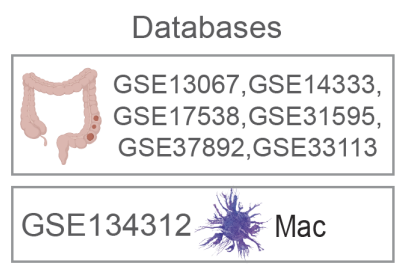

GSE63626 Fibroblast Boolean Implication

GSE24759 Oolymphocyte

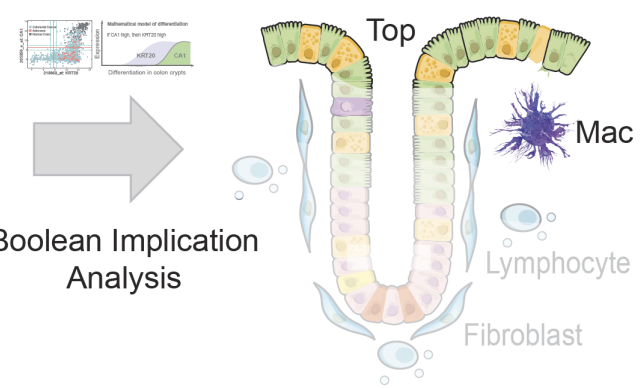

H

Predict Cell Type Specific Expression
M

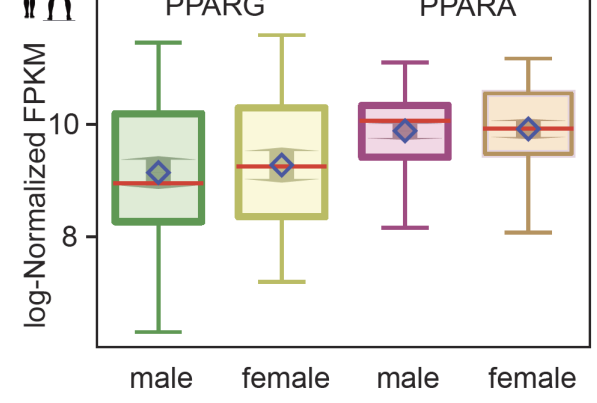

Figure 2: Network-guided rationalization of PPARA/PPARG as targets in IBD. (A) An interactive web-based platform allows the querying of paths of gene clusters in the IBD map [(9); see Supplementary Fig. 1] to pick high-value targets with a few mouse 

made available under aCC-BY 4.0 International license.

clicks and generate a comprehensive automated target 'report card'. The components of a 'target report card' is shown (right): predicted 'therapeutic index' (likelihood of Phase III success), IBD outcome (prognostic potential in UC and/or CD), networkprioritized mouse model, estimation of gender bias and predicted tissue cell type of action. (B-H) Components of a target report card for PPARA and PPARG are displayed. Bar plot (B; top) displays the rank ordering of normal vs. ulcerative colitis (UC)/Crohn's Disease (CD) patient samples using the average gene expression patterns of the two genes: PPARG/PPARA. Samples are arranged from highest (left) to lowest (right) levels. ROC-AUC statistics were measured for determining the classification strength of normal vs IBD. Bar plots (B; top) and violin plots (B; bottom) display the differences in the average expression of the two genes in normal, UC and CD samples in the test cohort that was used to build the IBD-map in (9). Bar plots in panel C-D show the rank ordering of either normal vs. IBD samples (C) or responder vs. non-responder (R vs. NR; D), or active vs. inactive disease, or neoplastic progression in quiescent $\mathrm{UC}$ (qUC $v s . \mathrm{nUC}$; D) across numerous cohorts based on gene expression patterns of PPARG and PPARA, from highest (left) to lowest (right) levels. Classification strength within each cohort is measured using ROC-AUC analyses. Bar plots in panel $\mathrm{E}$ show the rank ordering of either normal vs IBD samples across numerous published murine models of IBD based on gene expression patterns of PPARG and PPARA as in $\mathrm{D} . \mathrm{ACT}=$ adoptive $\mathrm{T}$ cell transfer. Classification strength within each cohort is measured using ROC-AUC analyses. Bulk = whole distal colon; epithelium = sorted epithelial cells. Schematic in F summarizes the computational prediction of the cell type of action for potential PPARA/G targeted therapy, as determined using Boolean implication analysis. GSEID\# of multiple publicly available databases of the different cell types and colorectal datasets used to make sure predictions are cited. Red boxes/circles denote that $P P A R A / G$-targeted therapeutics are predicted to work on monocytes/macrophages and crypt-top enterocytes. Computationally generated therapeutic index (see Methods) is represented as a line graph in $\mathrm{G}$. The annotated numbers represent Boolean implication statistics. PPARA and PPARG align with other targets of FDA approved drugs on the right of threshold (0.1). Two FDA approved targets (green; ITGB1, 0.046; JAK2, 0.032), two abandoned targets (red; SMAD7, 0.33; IL11, 0.16), PPARA (grey, 0.064), PPARG (grey, 0.04), and the threshold (black, 0.1) are shown in the scale. Box plot in panel $\mathrm{H}$ shows that the level of PPARA/G expression is similar in the colons of both genders in health and in IBD, and hence, PPAR $\alpha / \gamma$-targeted therapeutics are predicted to have little/no gender predilection. 

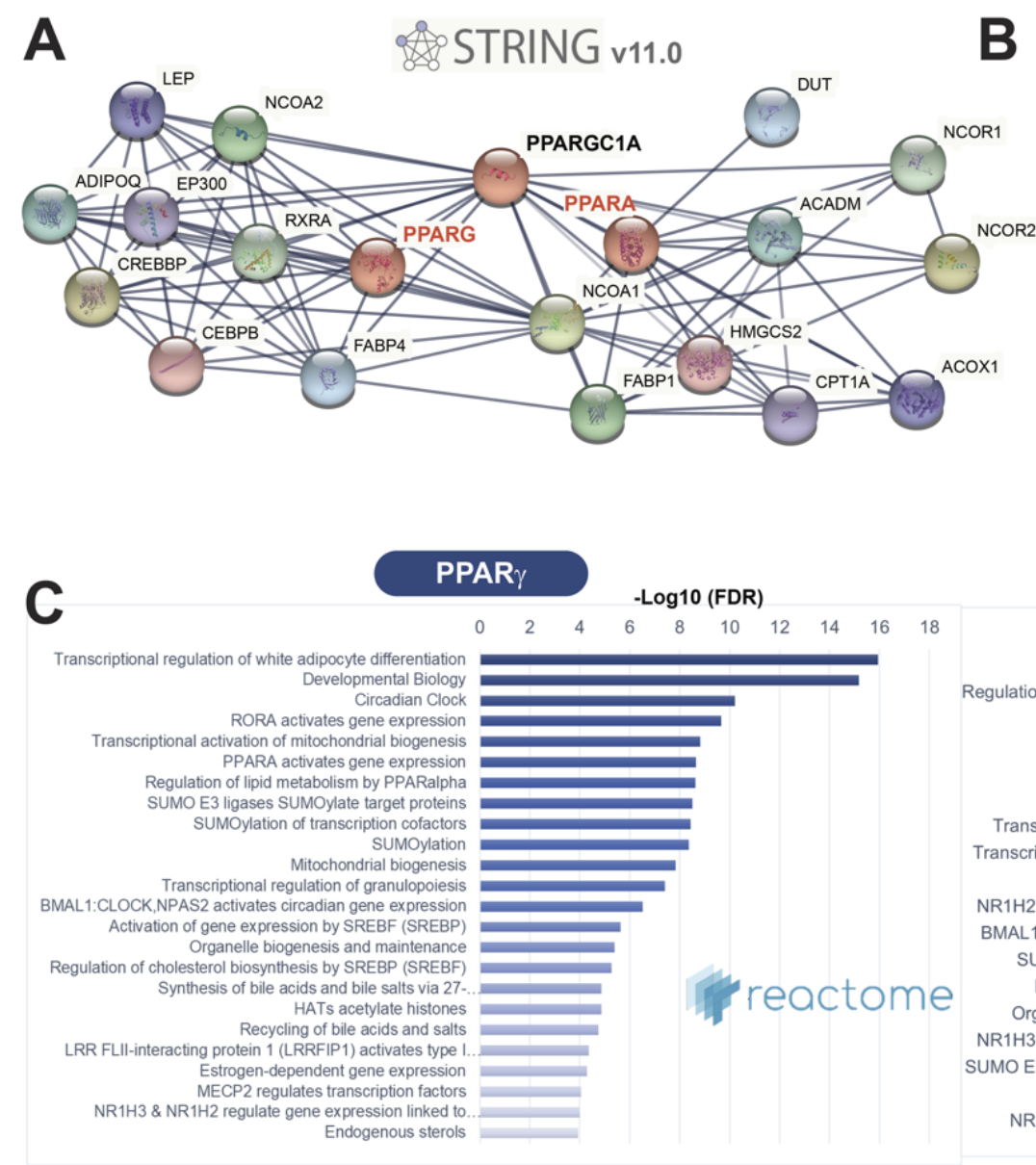

B

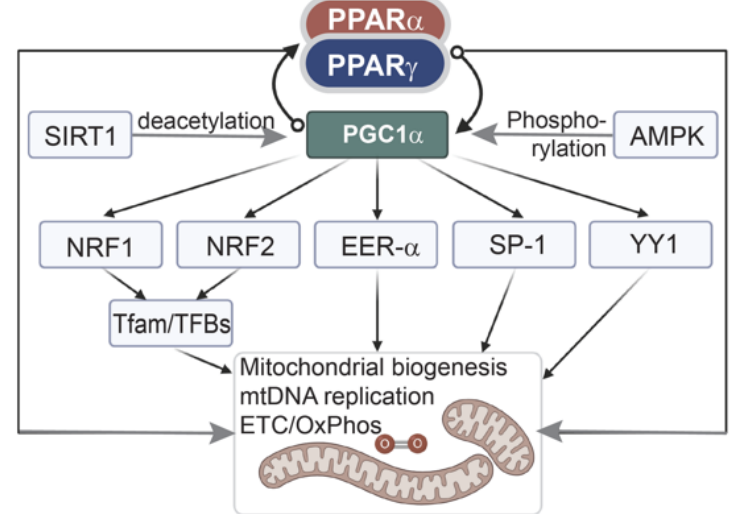

PPAR $\alpha$
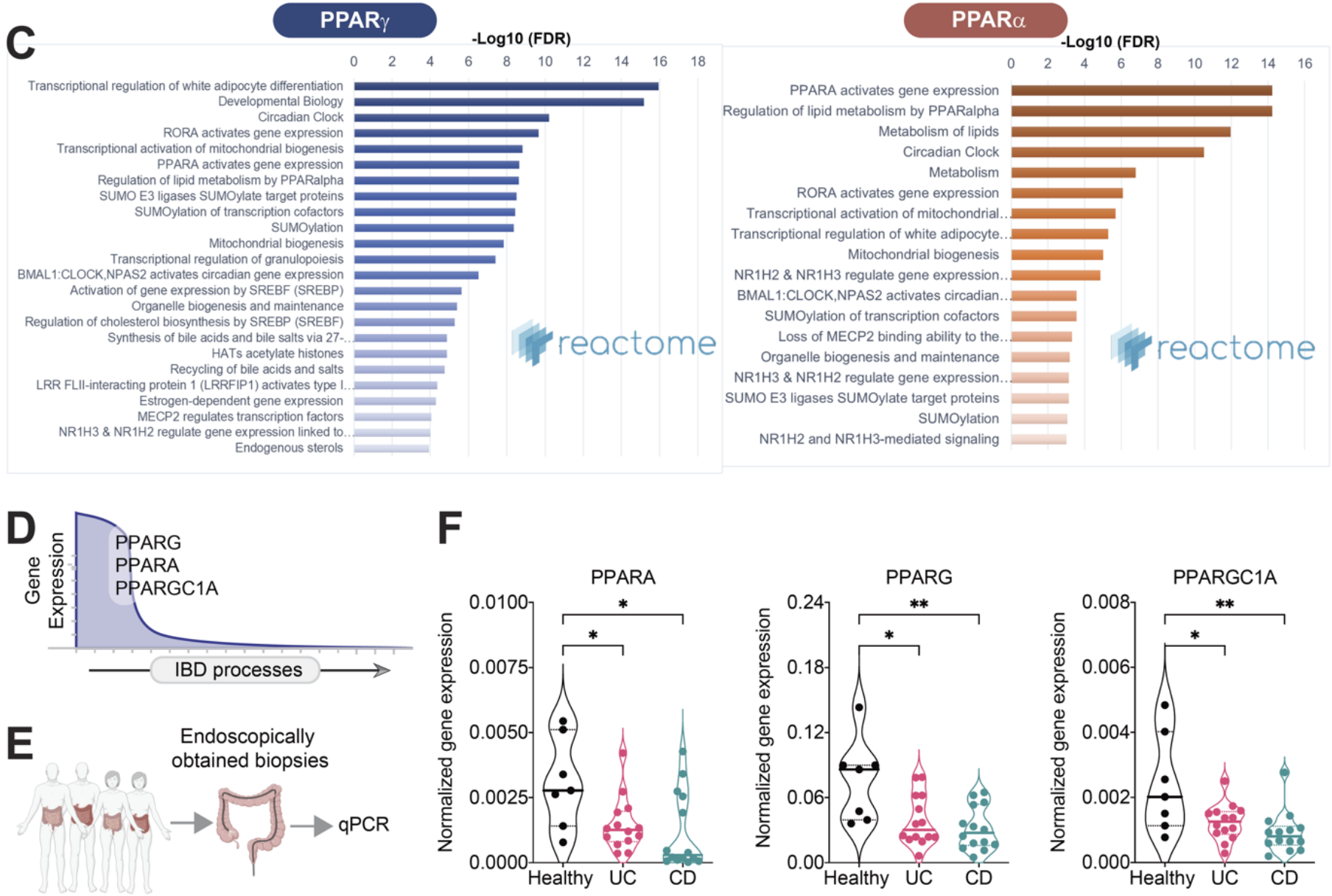

7

8

Figure 3: Rationalization of PPAR $\alpha$ and PPAR $\gamma$ as targets in IBD.

(A) A protein-protein interaction network (i.e., interactomes) for PPAR $\alpha$ and PPAR $\gamma$, generated using STRING v.11 (https://stringdb.org). (B) Schematic summarizing the roles of PPAR $\alpha$, PPAR $\gamma$ and PGC1 $\alpha$ on mitochondria biogenesis and function (based on). PGC1- $\alpha$ emerges as a critical hub for forward feedback loops. (C) Reactome pathway analyses (www.reactome.org) on PPAR- $\alpha$ and PPAR- $\gamma$ interactomes in A show convergence on metabolism, mitochondria bioenergetics and the circadian clock. (D) Graphical visualization of the predicted changes in the expression of PPARA (PPAR- $\alpha$ ), PPARG (PPAR- $\gamma$ ) and PPARGC1A (PGC1- $\alpha$ ) genes during the progression of IBD processes (indicated with an arrow). (E) Schematic showing validation workflow; the expression of PPARA, PPARG and PPARGC1A transcript levels were assessed in the ileum/colon biopsies of IBD patients $(\mathrm{UC}=14$ and $\mathrm{CD}=$ 14)) or healthy controls ( $\mathrm{n}=7)$. (F) Violin plots display the qPCR results in E. Results are displayed as mean \pm SEM. Significance was tested using one-way ANOVA followed by Tukey's test for multiple comparisons. Significance: ${ }^{*}, \mathrm{p}<0.05 ; * *, \mathrm{p}<0.01$. 
bioRxiv preprint doi: https://doi.org/10.1101/2021.07.18.452807; this version posted July 19, 2021. The copyright holder for this preprint (which was not certified by peer review) is the author/funder, who has granted bioRxiv a license to display the preprint in perpetuity. It is made available under aCC-BY 4.0 International license.

A

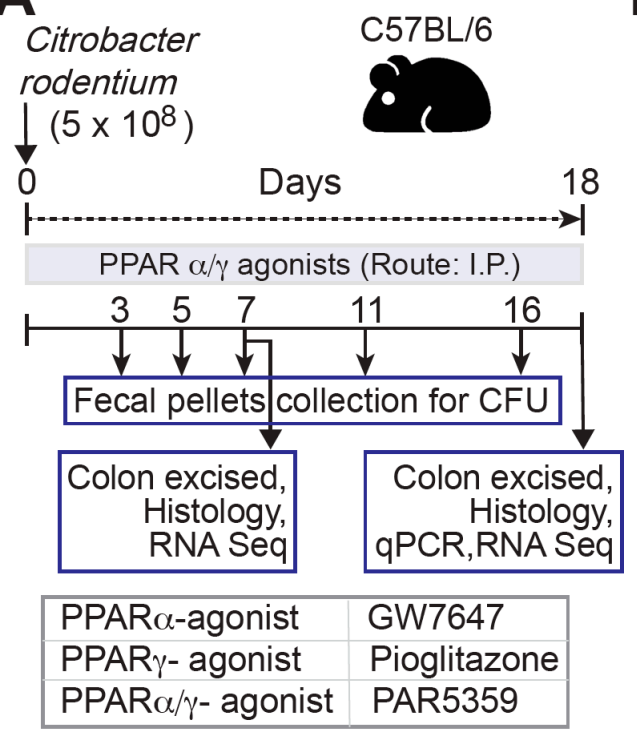

B

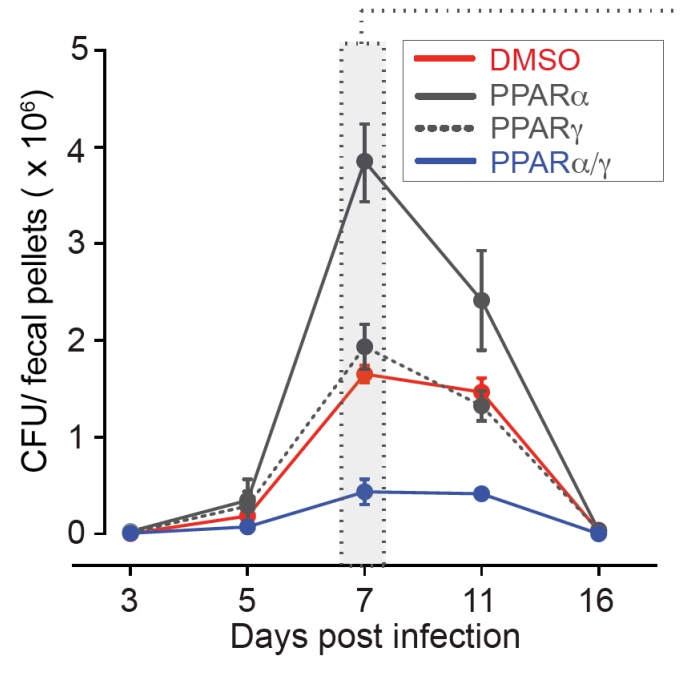

C $\mathrm{CFU} / \mathrm{\ddots}$ fecal pellets (day \#7)

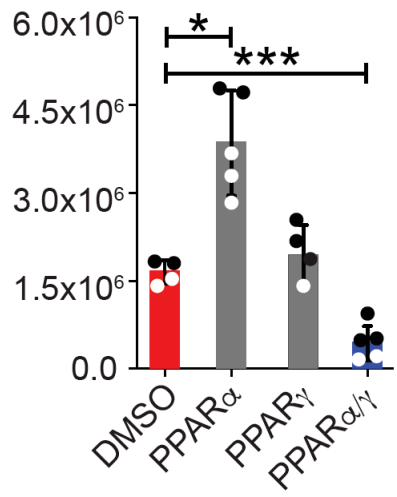

D

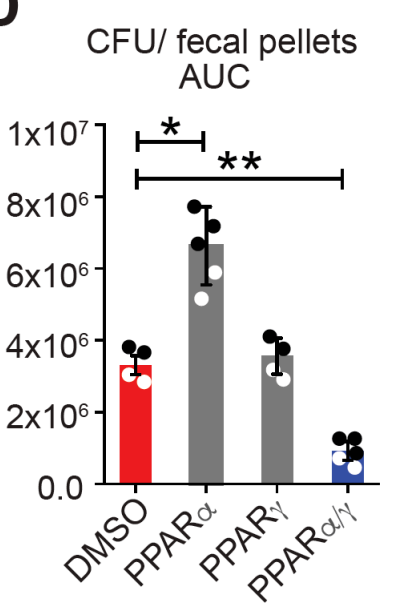

\section{E DMSO}

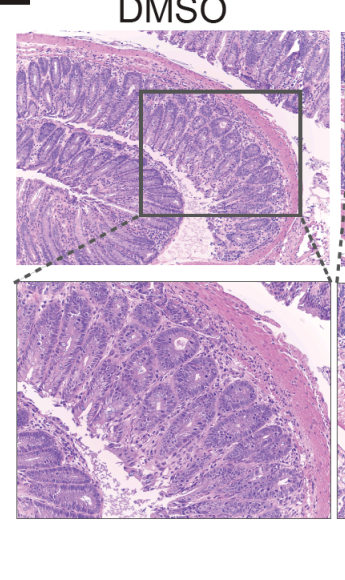

PPAR $\alpha$

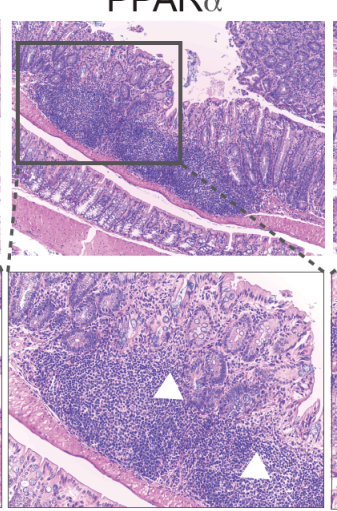

PPARy
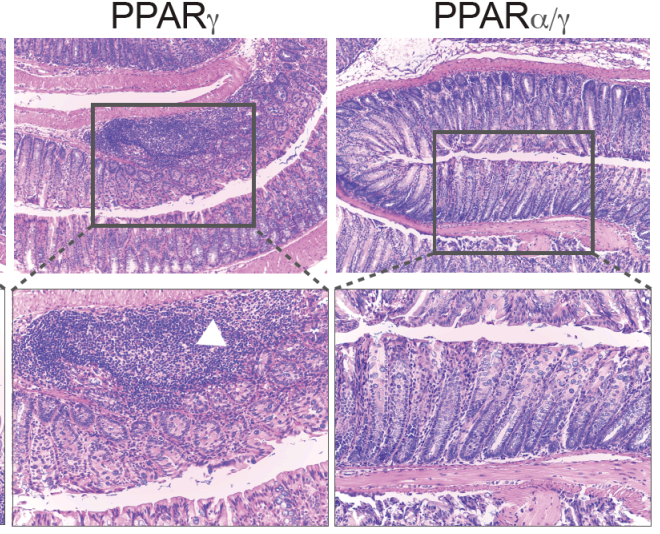

$\mathbf{F}$

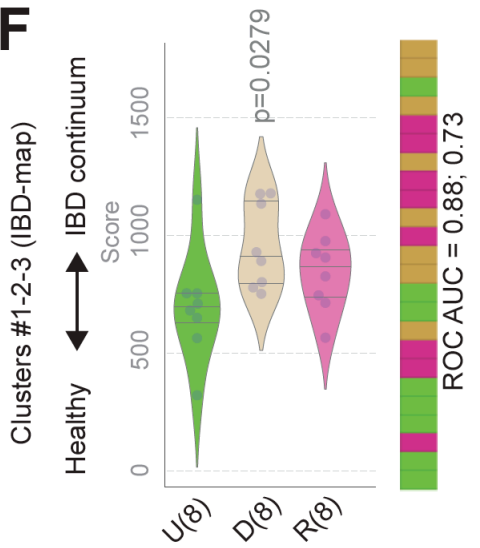

G

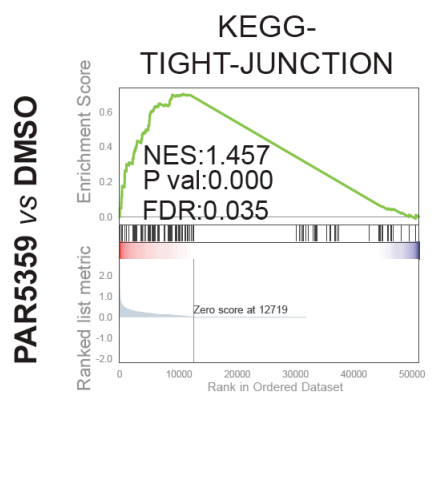

GO-ADHERENS-

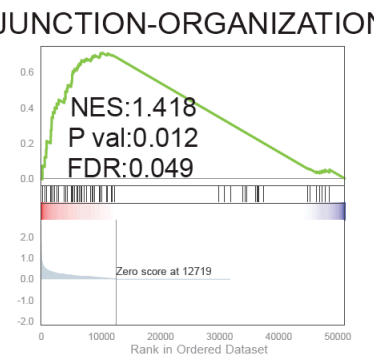

COATES-MACROPHAGE-

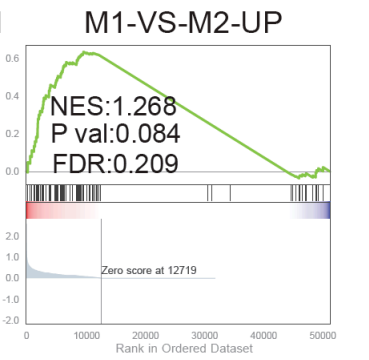

Uninfected $\square$ DMSO $\square$ PAR-5359

Figure 4: PPAR $\alpha / \boldsymbol{\gamma}$ dual agonists ameliorate Citrobacter rodentium-induced infectious colitis in mice.

(A) Schematic summarizing the workflow for testing PPAR-targeted therapeutics in C. rodentium-induced colitis. Mice were gavaged with $C$. rodentium on day 0 and subsequently treated daily with PPAR agonists. Fecal pellets were collected to test viable bacterial burden, as determined by dilution plating and colony counting. Colons were excised on day 7 and 18 and analyzed using the indicated readouts. (B-D) Line graphs in B display time series of the burden of viable bacteria in feces. Scatter plots with bar graphs in $\mathrm{C}$ compare the peak burden of viable bacteria in feces on day 7. Scatter plots with bar graphs in D display the area under the curve (AUC) for the line graph in B. (E) Images display representative fields from H\&E-stained colon tissues. Mag = 100x (top) 
1 and 200x (bottom). White arrowheads point to immune cell infiltrates. Statistics: All results are displayed as mean \pm SEM.

2 Significance was tested using two-way/one-way ANOVA followed by Tukey's test for multiple comparisons. Significance: *, p $<$ $30.05 ; * *, p<0.01, * * *, p<0.001$. (F) Violin plots (left) display the deviation of expression of genes in Clusters \#1-2-3 in the IBD 4 network, as determined by RNA Seq on murine colons. Bar plot (right) displays the rank ordering of the samples. (G) Pre-ranked 5 GSEA based on pairwise differential expression analyses (DMSO vs PAR5359 groups) are displayed as enrichment plots for 6 epithelial tight (left) and adherens (middle) junction signatures and balanced macrophage processes (right). See also Supplemental 7 Figure 7 for the Day \#7 results in the $C$. rodentium-induced colitis model, Supplemental Figure 8 for extended GSEA analyses, 8 and Supplemental Figure 9 for the effect of PAR5359 on DSS-induced colitis in mice. 
A

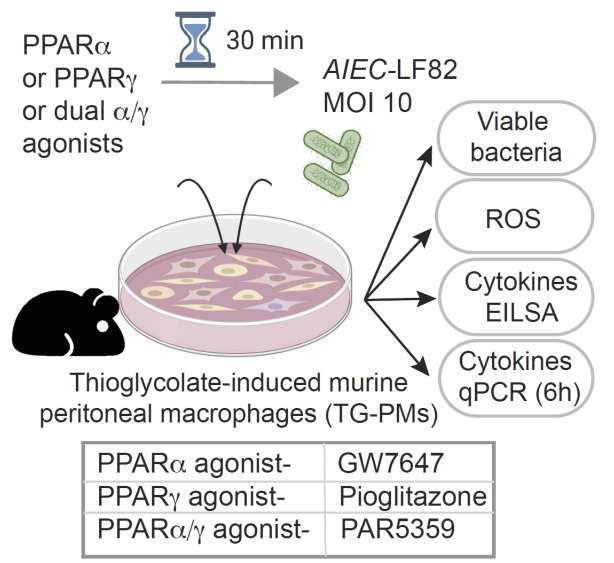

B
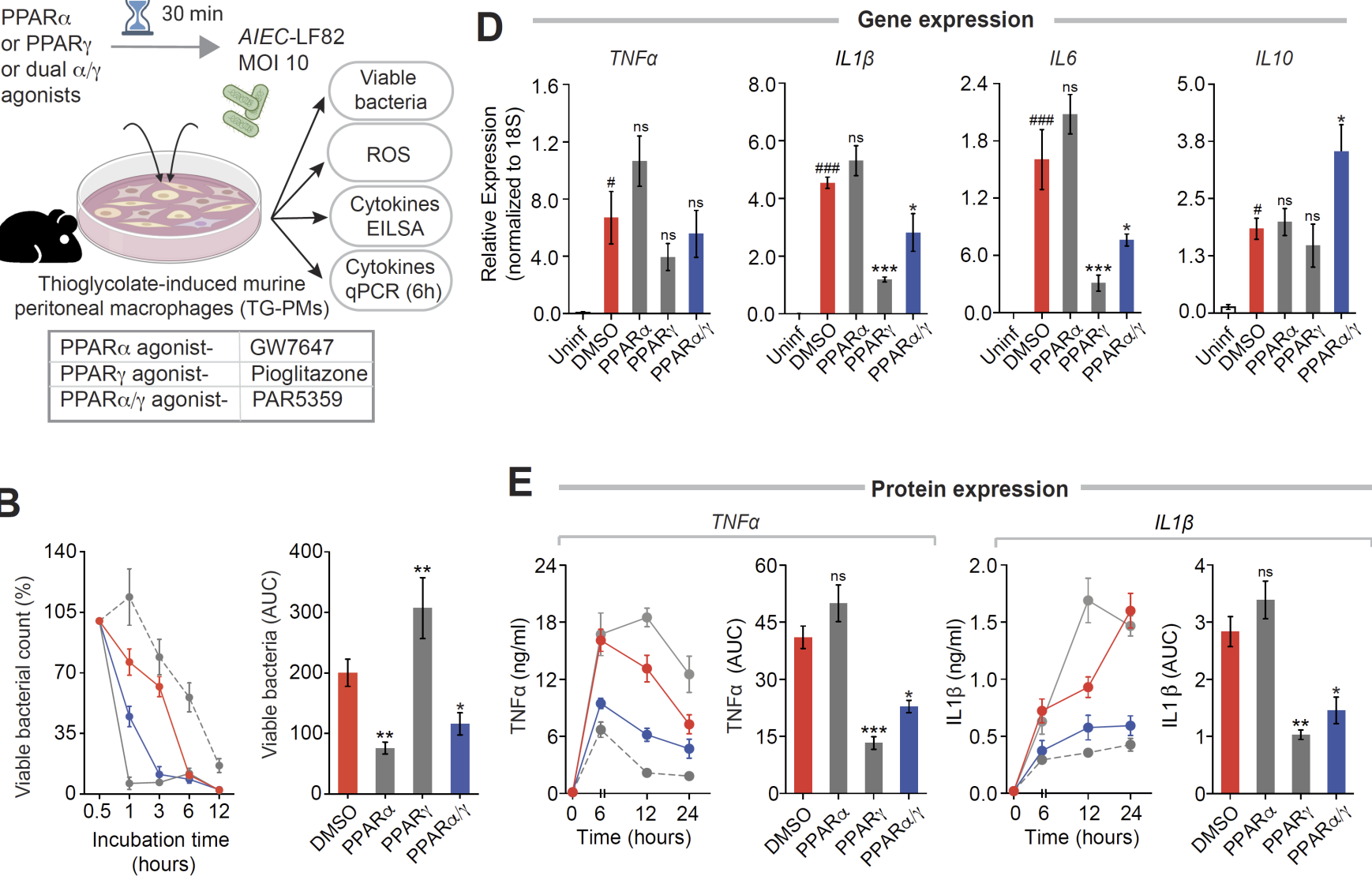

$E$

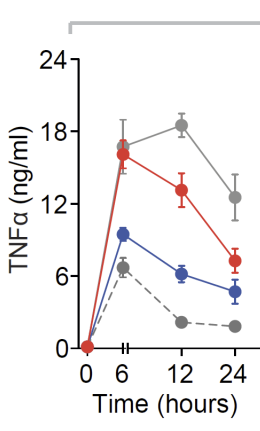

$T N F \alpha$

Protein expression
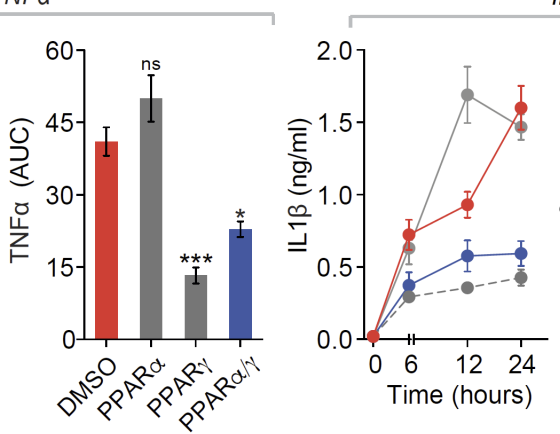

IL1ß

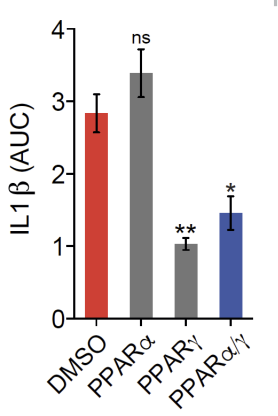

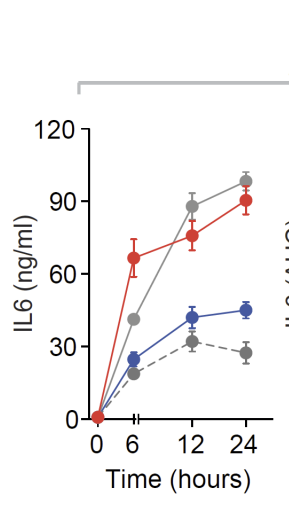
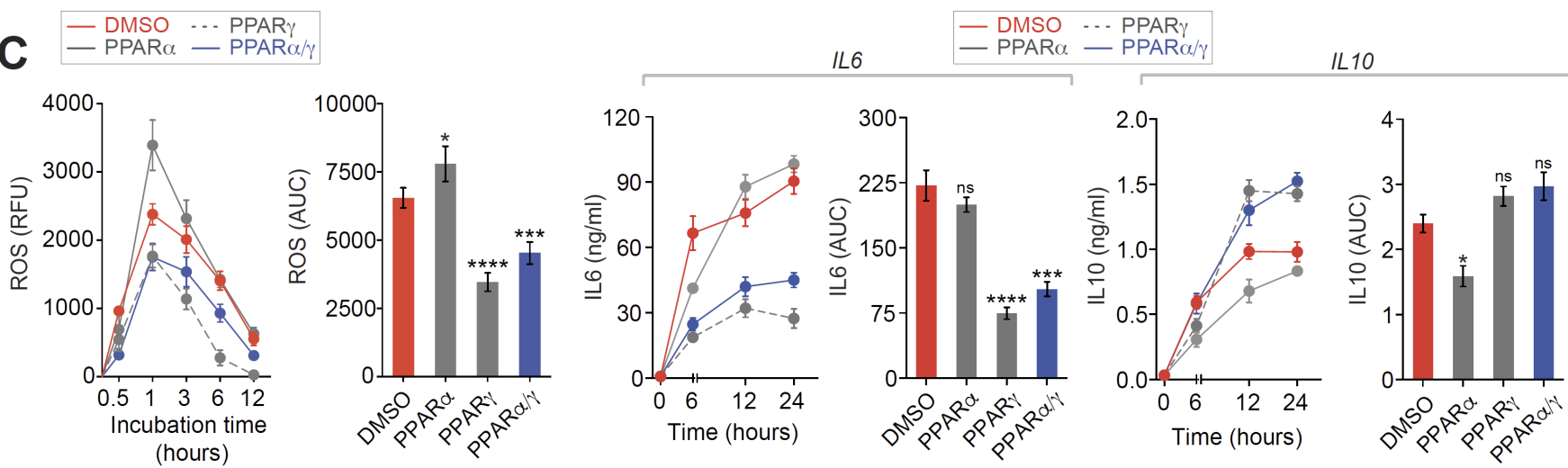

Figure 5: PPAR $\alpha$ and PPAR $\alpha / \gamma$-dual agonists enhance, whereas PPAR $\gamma$ agonist delay bacterial (AIEC-LF82) clearance.

(A) Schematic displays the experimental design and workflow. Thioglycolate-induced murine peritoneal macrophages (TG-PM) pretreated with PPAR agonists (see box, below; $20 \mathrm{nM} \mathrm{GW7647,} 10 \mu \mathrm{M}$ Pioglitazone and $1 \mu \mathrm{M}$ PAR5359) were infected with AIEC-LF82 (MOI 10) and subsequently analyzed for the bacterial count (Gentamicin protection assay), generation of cellular ROS, secretion of inflammatory cytokines (in supernatant media by ELISA) and the induction of cytokines (gene transcript analysis by qPCR). (B) Line graphs (left) display percent viable bacterial counts at indicated times after infection. Bar graphs (right) display the AUC. (C) Line graphs (left) and bar graphs (right) display the extent of ROS generation over time. (D) Bar graphs display the relative expression of transcripts of multiple cytokines (IL1 $\beta$, IL6, TNF $\alpha$ and IL10). (E) Line graphs (left) and bar graphs (right) showing the levels of secreted cytokines in the media. Statistics: All results are from at least three independent experiments and results displayed as means \pm SEM. Significance was tested using two-way/one-way ANOVA followed by Tukey's test for multiple comparisons. Significance: '\#’ significance over uninfected TG-PMs and '*' shows significance over AIEC-LF82 infected cells. ns, non-significant, *, p $<0.05$ * $^{* *}, \mathrm{p}<0.01,{ }^{* *}, \mathrm{p}<0.001,{ }^{* * *}, \mathrm{p}<0.0001$. See Supplemental Figure 10 for similar bacterial clearance assays performed using Salmonella enterica. 


\section{A}

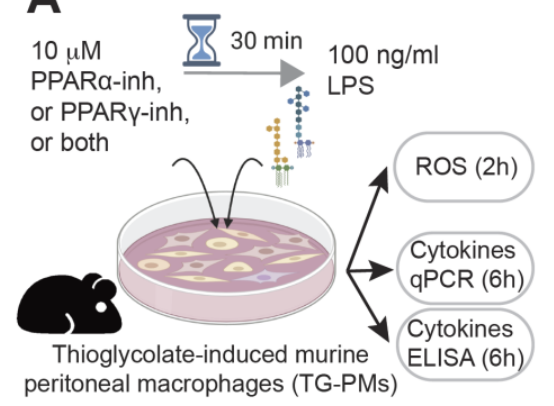

C
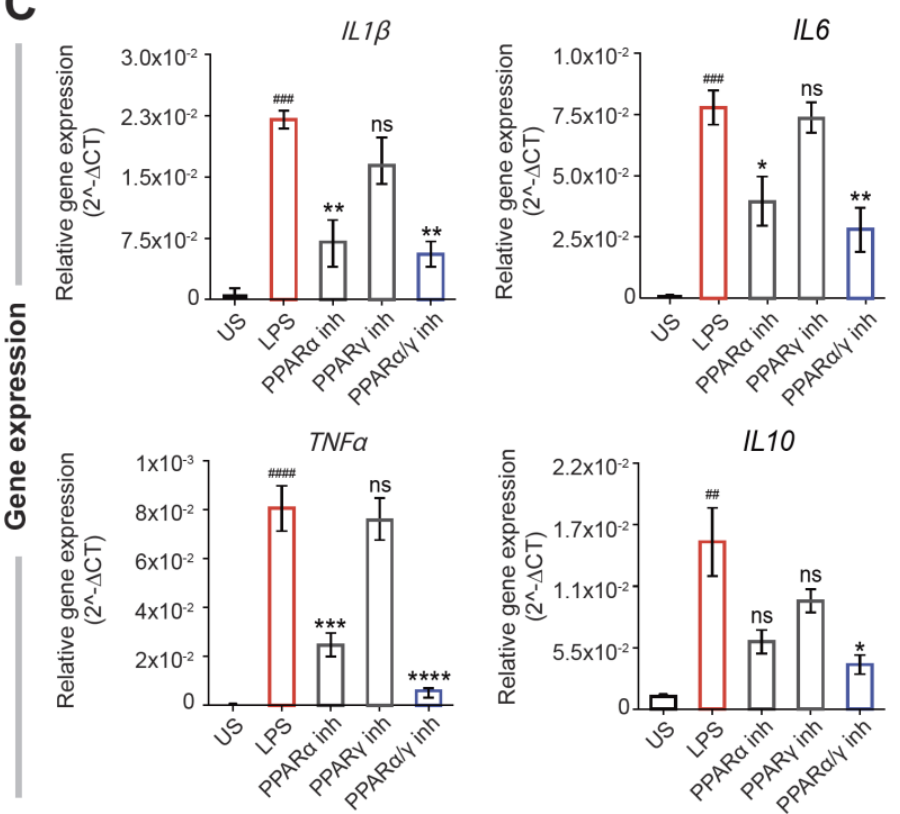
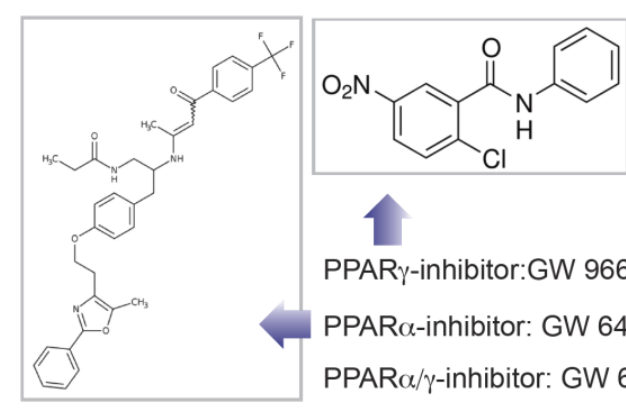

PPAR $\gamma$-inhibitor:GW 9662

PPAR $\alpha$-inhibitor: GW 6471

PPAR $\alpha / \gamma$-inhibitor: GW 6471+GW 9662

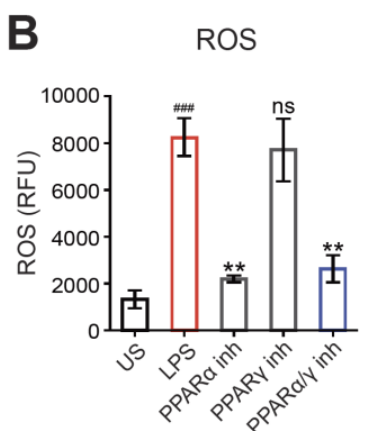

D

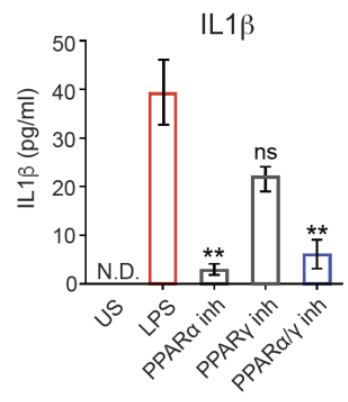

TNF $\alpha$

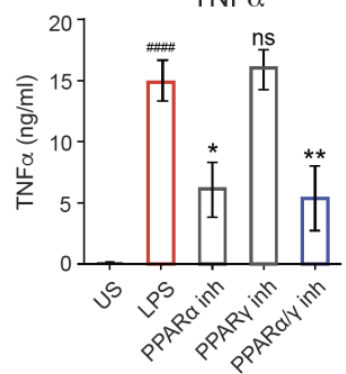

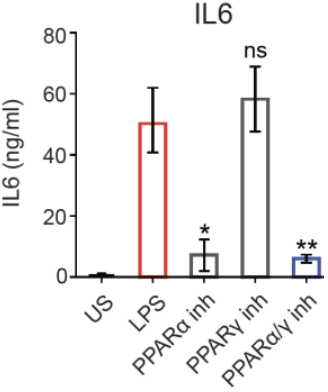

IL10

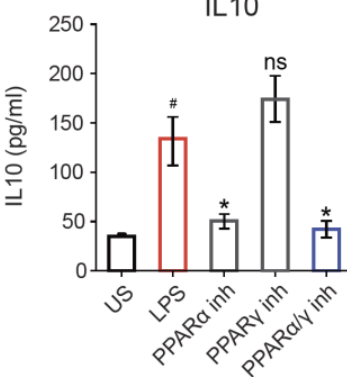

Figure 6: PPAR $\alpha$ but not PPAR $\gamma$ is required for induction of cellular ROS and proinflammatory cytokines.

(A) Schematic of experimental design. TG-PMs were pre-incubated with $10 \mu \mathrm{M}$ PPAR $\alpha$ or PPAR $\gamma$ inhibitors, either alone or in combination for $30 \mathrm{~min}$ prior to stimulation with $100 \mathrm{ng} / \mathrm{ml}$ LPS. Cells were analyzed at 2 and 6 h to estimate cellular ROS and cytokine induction, respectively. (B-D) Bar graphs display the levels of cellular ROS (B), relative levels of mRNA (C) and protein (D) expression of cytokines (IL1 $\beta$, IL6, TNF $\alpha$ and IL10). Statistics: Results are from three independent experiments and displayed as mean \pm SEM. One-way ANOVA followed by Tukey's test for multiple comparisons was performed to test significance. Significance: ns: non-significant, ${ }^{*}, \mathrm{p}<0.05 ; * *, \mathrm{p}<0.01, * * *, \mathrm{p}<0.001$ and $* * * *, \mathrm{p}<0.0001$. 

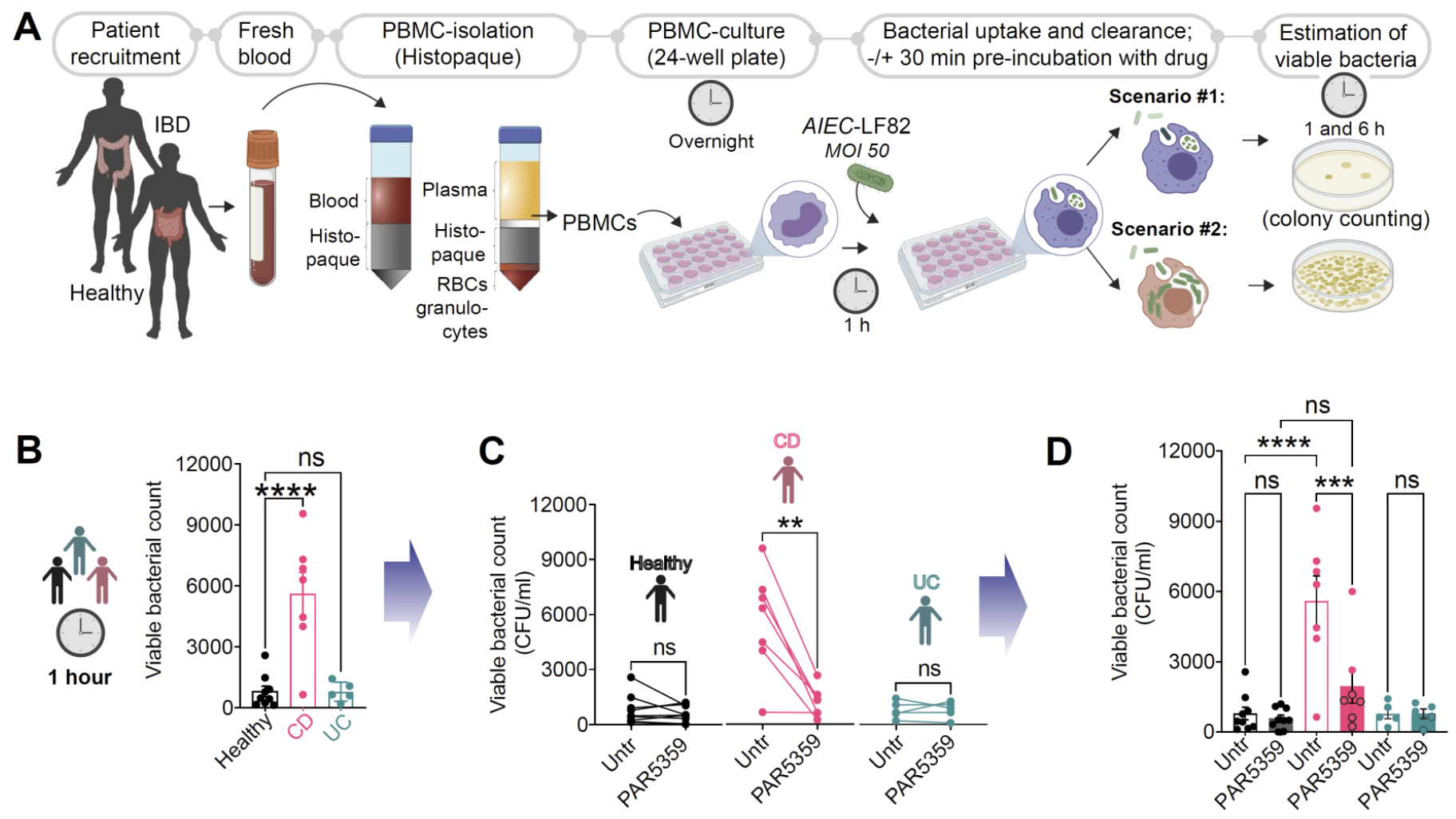

E

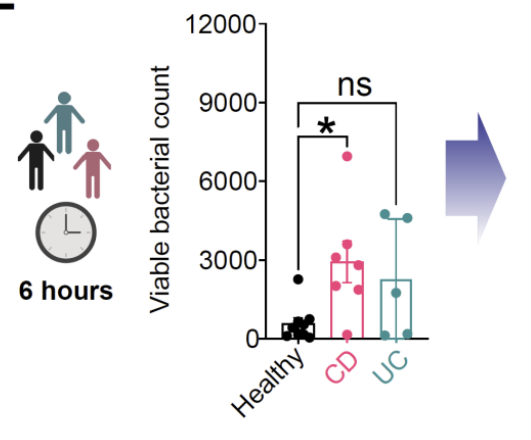

$\mathbf{F}$

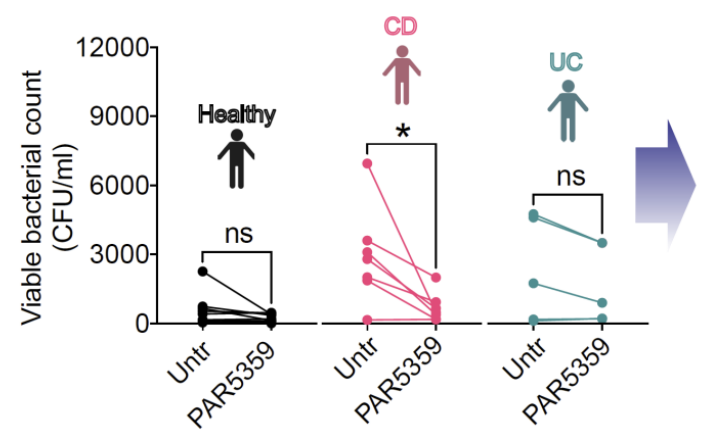

G

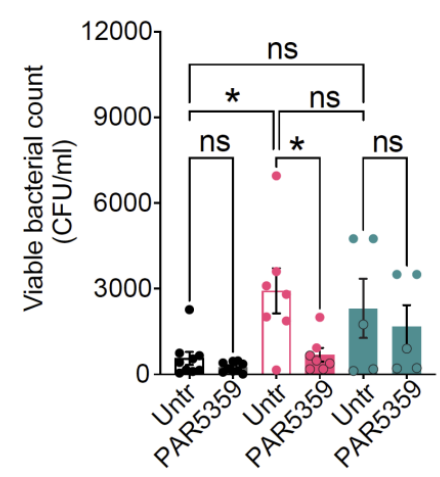

Figure 7: PPAR $\alpha / \gamma$ dual agonist, PAR5359, promotes the clearance of $A I E C$-LF82 from CD patient-derived PBMCs. (A) Schematic displays the overall experimental design using human subjects (see Supplemental Table 5 for patient demographics). Peripheral blood collected from healthy, CD and UC patients was used as a source of PBMCs. PBMCs were pre-treated for $30 \mathrm{~min}$ with $1 \mu \mathrm{M}$ PPAR $\alpha / \gamma$ agonists prior to infection with AIEC-LF82 (MOI 50) for $1 \mathrm{~h}$. PBMCs were subsequently treated with gentamicin to kill extracellular microbes for $60 \mathrm{~min}(\sim \mathrm{t} 0 \mathrm{~h})$ prior to lysis and plating to determine the intracellular abundance of viable bacteria at $\mathrm{t} 1 \mathrm{~h}$ and $\mathrm{t} 6 \mathrm{~h}$, as determined by dilution plating and colony counts (see Methods for details). Bar graphs with scatter plot display the abundance of viable intracellular bacteria at $1 \mathrm{~h}(\mathbf{B})$ and $6 \mathrm{~h}(\mathbf{E})$ after infection. Paired line plots display the rate of clearance of bacteria in individual subjects at $1 \mathrm{~h}(\mathbf{C})$ and $6 \mathrm{~h}(\mathbf{F})$ after infection. Data in B-C of $1 \mathrm{~h}$ infection is combined in (D) and data from $\mathrm{E}$ and $\mathrm{F} 6 \mathrm{~h}$ infection is combined in (G) with statistics: Results are displayed as mean \pm SEM (CD patient $\mathrm{n}=7$, UC patients $=6$ and healthy $n=9$ ). Paired t-test or One-way ANOVA followed by Tukey's test for multiple comparisons was performed to test significance. Significance: ns: non-significant, ${ }^{*}, \mathrm{p}<0.05 ;{ }^{* *}, \mathrm{p}<0.01,{ }^{* * *}, \mathrm{p}<0.001$ and $* * * *, \mathrm{p}<0.0001$. 


\section{Network-rationalized target identification}

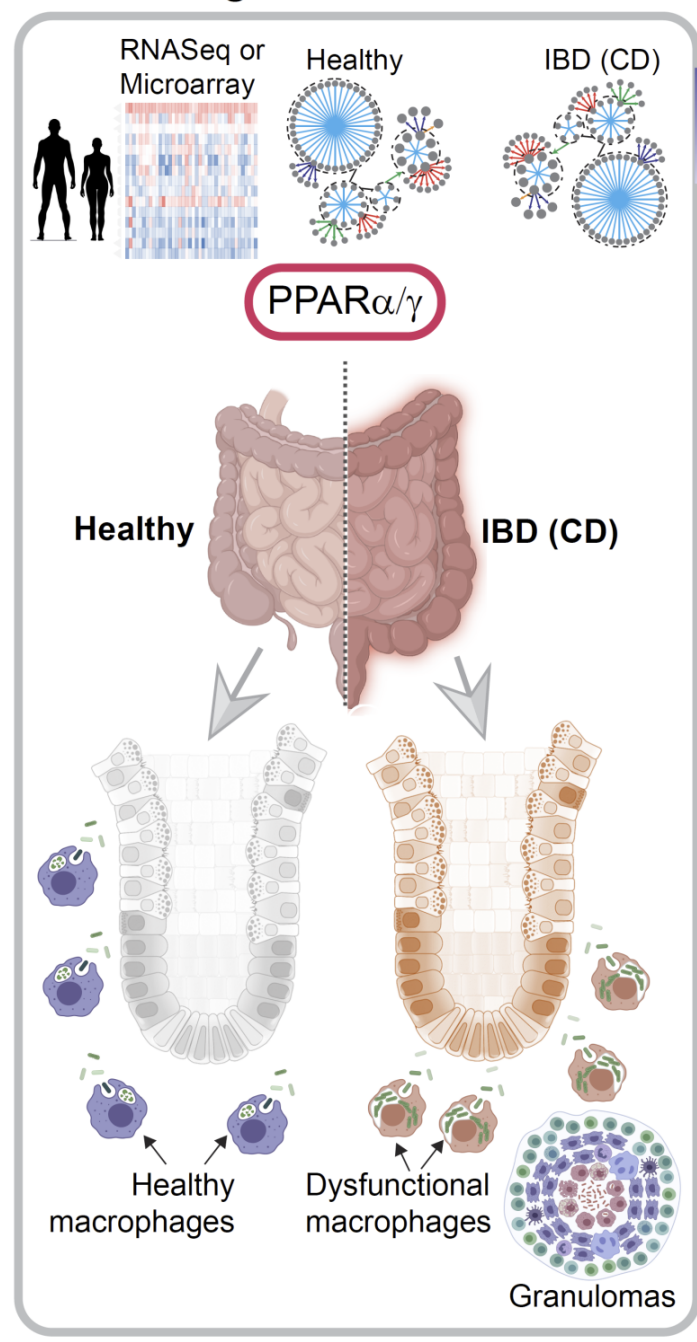

\section{Experimental determination of} mechanism of action

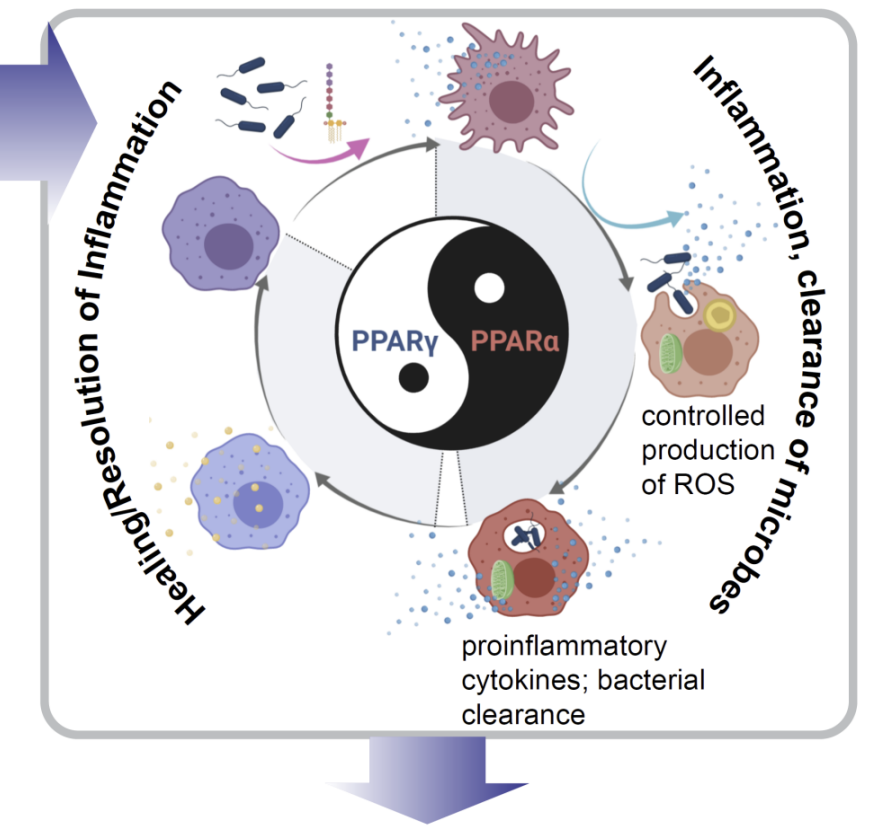

Validation in pre-clinical models

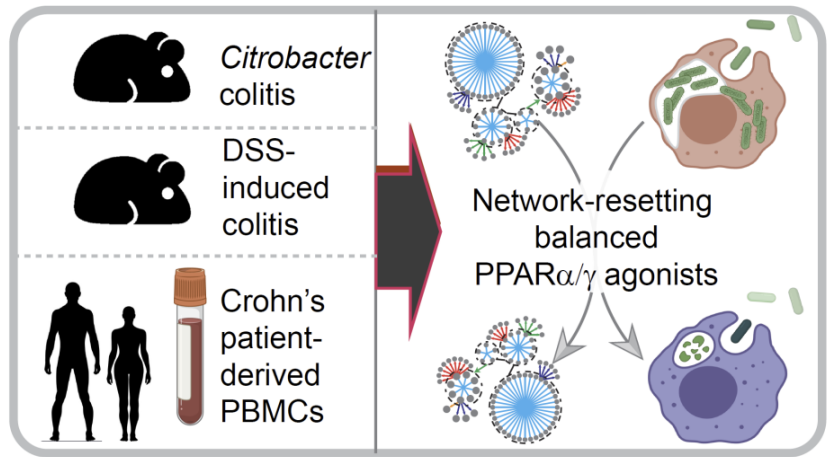

Figure 8: Summary of findings and working model

Schematic summarizes key approaches and findings of this study. First, network-rationalized target identification (Left) was performed using web-based platform that queries > 1000 IBD datasets [(9); see Methods] that served as 'input' to create a map of gene clusters that are progressively altered in the gut in the setting of IBD. Predictions are used to guide the choice of therapeutics (dual agonists of PPAR $\alpha$ and PPAR $\gamma$ that have a balanced agonistic potential for both PPARs), the choice of animal models of IBD, predict cell types of action (macrophage processes), and finally, the subtype of IBD that could benefit most based on the cell type of action (i.e., CD). Second, experimentally determined mechanism of action studies (right, top) showed that balanced actions of both PPAR $\alpha$ and PPPAR $\gamma$ enable the induction of bacterial clearance, resolution of inflammation and healing; PPAR $\alpha$ is responsible for ROS and cytokine induction, whereas PPPAR $\gamma$ is responsible for anti-inflammatory response and healing. The dual agonistic action was superior to each agonist used alone. Third, targets validation studies (right, bottom) in murine and human models confirm the use of PPAR $\alpha / \gamma$ dual agonists for enhancing bacterial clearance and protection against colitis. When tested side-by-side in the infectious colitis model, the dual agonistic action was superior to each agonist used alone. 